\title{
Emplazamiento del magmatismo Paleoceno-Eoceno bajo un régimen transtensional y su evolución a un equilibrio dinámico en el borde occidental de Colombia
}

\author{
Jeny Alejandra Grajales ${ }^{1,2, *}$, Ángel F. Nieto-Samaniego ${ }^{3}$, \\ Darío Barrero Lozano 4 , Jairo Alonso Osorio ${ }^{5}$ y \\ Mario Andrés Cuellar 5 \\ ${ }^{1}$ Universidad de Concepción, Facultad de ciencias Químicas, \\ Edmundo Larenas 234, Campus Concepción, Chile. \\ ${ }^{2}$ La Luna E\&P S DE RL Sucursal Colombia, Carrera 13A No. 87-17, Bogotá, Colombia. \\ ${ }^{3}$ Centro de Geociencias, Universidad Nacional Autónoma de México, Campus Juriquilla, \\ Boulevard Juriquilla 3001, C.P. 76230, Querétaro, México. \\ ${ }^{4}$ Consultor independiente. Calle 113 No. 11A-55, Bogotá, Colombia. \\ ${ }^{5}$ Servicio Geológico Colombiano, Diagonal 53, No. 34 - 53, Bogotá D.C., Colombia. \\ *Grajales.jennyalejandra@gmail.com
}

\section{RESUMEN}

Los arcos magmáticos del Cretácico y Paleógeno de las Cordilleras Central y Occidental de Colombia han sido atribuidos a la evolución del sistema de subducción de la costa pacífica colombiana. En esa región se han propuesto sucesivas acreciones de terrenos, configurando la estructura de ambas cordilleras. En este trabajo se analiza la distribución y las edades de cristalización de plutones emplazados entre 60 Ma y $53 \mathrm{Ma}$ en las Cordilleras Central y Occidental. A partir de los $53 \mathrm{Ma}$, el arco magmático migró hacia el occidente colombiano, donde se instaló en una corteza de borde continental. Las edades de cristalización analizadas sugieren que dentro del área de estudio el cinturón de plutones es continuo a lo largo de la cordillera Occidental. De $40 \pm 5 \mathrm{Ma}$ a $26 \mathrm{Ma}$ se dio una disminución significativa en la velocidad de convergencia de la placa Farallón, lo que dio lugar a un proceso de relajación del esfuerzo regional, al reducirse la carga tectónica. El proceso de relajación del esfuerzo regional también fue reflejado en los ambientes intra-continentales, dando lugar a procesos de peneplanización en los altos topográficos del norte colombiano, la reactivación del piedemonte cordillerano con progradaciones de la sedimentación hacia el occidente y el desarrollo de la discordancia regional del Eoceno medio/tardío. En los ambientes de plataforma continental se reflejó por la distribución de secuencias de calizas arrecifales a lo largo de la margen pacífica colombiana, del caribe de Colombia, Ecuador, Panamá y Costa Rica, y finalmente, por un gap magmático comprendido entre $33 \mathrm{Ma} \mathrm{y} 26 \mathrm{Ma}$. El evento magmático Paleoceno - Eoceno distribuido en las cordilleras Central y Occidental del occidente colombiano fue emplazado en un régimen tectónico transtensional, con el esfuerzo compresivo horizontal máximo $\left(\sigma_{1}\right)$ en dirección SW-NE, producto de la convergencia oblicua entre las placas Farallón y Sudamericana.

Palabras clave: Régimen transtensional; convergencia oblicua; emplazamiento magmático; migración del arco; equilibrio dinámico.

\begin{abstract}
The Cretaceous and Paleogene magmatic arcs of the Central and Western Cordilleras of Colombia have been attributed to the evolution of a subduction system in the Colombian Pacific coast. In this work the distribution and crystallization ages of plutons emplaced between $60 \mathrm{Ma}$ and $53 \mathrm{Ma}$ in the Central and Western Cordilleras are analyzed.

From 53 Ma the magmatic arc migrates towards the west of Colombia, installing magmas in a plate edge transitional crust. The crystallization ages analyzed in this work suggest that, within the study area, the plutonic belt is continuous throughout the Western Cordillera. From $40 \pm 5 \mathrm{Ma}$ to $26 \mathrm{Ma}$ there was a significant reduction in the convergence velocity of the Farallon plate; as it decreases, also the tectonic loading diminishes resulting in a process of regional stress relaxation. The process of relaxation of the regional stress also occurred in the intra-continental environments producing peneplanization process in the topographic highs of northern Colombia, the reactivation of the piedmont with westwards progradation of sedimentation and the development of a middle- to late-Eocene regional unconformity. In continental shelf environments, the relaxation of the tectonic stress is evidenced by the distribution of reef limestone sequences throughout the Colombian Pacific margin and
\end{abstract}


the Caribbean of Colombia, Ecuador, Panama and Costa Rica, and by a magmatic gap from $33 \mathrm{Ma}$ to $26 \mathrm{Ma}$. The Paleocene-Eocene magmatic event distributed in the Central and Western Cordilleras took place under a transtensional regime, with the maximum horizontal compressive stress $\left(\sigma_{1}\right)$ oriented $S W-N E$, product of the oblique convergence between the Farallon and South American plates.

Key words: Transtensional regime; oblique convergence; magmatic emplacement; arc migration; dynamic balance.

\section{INTRODUCCIÓN}

Los arcos magmáticos en el borde occidental de Colombia han sido objeto de estudio en las últimas décadas. Fueron descritos por primera vez por Aspden et al. (1987). Esos eventos han sido atribuidos a la evolución de un sistema de subducción (Barrero, 1979; McCourt et al., 1984; Aspden et al., 1987; Grosser, 1989; Boschman et al., 2014) y a sucesivas acreciones de terrenos oceánicos al continente, bajo regímenes tectónicos compresivos y transpresivos de primer orden. (Duque-Caro, 1990; Estrada, 1995; Cediel et al., 2003; Pindell et al., 2005; Lissina, 2005; Ramos, 2009; Pindell y Kennan, 2009; Villagómez et al., 2011; Montes et al., 2012; Bayona et al., 2012; Spikings et al., 2014; Whattam y Stern, 2014; Boschman et al., 2014; Cardona et al., 2018; Montes et al., 2019).

En la Cordillera Central (CC) (Figura 1a) el magmatismo CretácicoPaleoceno tiene, principalmente, edades de cristalización entre $99 \mathrm{Ma}$ y $58 \mathrm{Ma}$ (Saenz et al., 1996; Restrepo et al., 2009; Leal Mejía, 2011; Bayona et al., 2012; Villagómez y Spiking, 2013; Jaramillo et al., 2017; Cardona et al., 2020) y ha sido asociado a la subducción oblicua de la placa Farallón bajo Sudamérica y a procesos pos-colisionales generados por la subducción de la Placa Caribe (Villagómez y Spikings, 2013; Jaramillo et al., 2017; Montes et al., 2019). Sobre la Cordillera Occidental (CO) (Figura 1a) este magmatismo se ha reportado con edades desde el Coniaciano - Maastrichtiano, asociándolo a procesos extensionales en el sistema de subducción (Barrero, 1979). En trabajos recientes y a partir de análisis geoquímicos y paleomagnéticos, el magmatismo Cretácico-Paleoceno (o incluso abarcando hasta el Eoceno) es considerado intraoceánico, acrecionado al continente durante el Mioceno como parte integral del Bloque Panamá-Chocó (Duque-Caro, 1990; Cediel et al., 2003; Ramos, 2009; Bayona et al., 2012; Pindell et al., 2005; Villagómez et al., 2011; Cardona et al., 2018; Montes et al., 2019) (Figura 1b). Esta hipótesis ha sido bien acogida en la literatura, aunque no se tengan estudios detallados de los límites en el borde continental, donde se propone que se acrecionaron dichos terrenos. Esos límites tectónicos han sido atribuidos a fallas transcurrentes, como fue propuesto por Duque-Caro (1990), quien sugirió la falla de Uramita como la sutura occidental, y la zona de deformación de Istmina, ubicada entre las fallas Garrapatas e Istmina, como la sutura sur (Duque-Caro, 1990 y Cediel et al., 2003). Sin embargo, hasta la fecha no se han documentado características geológicas, geométricas y cinemáticas que permitan definirlas como zonas de sutura de la acreción del Bloque Panamá-Choco. Los estudios publicados sobre el origen y evolución de los arcos magmáticos Paleoceno-Eoceno no consideran, en el borde continental colombiano, la continuidad espacial y temporal del arco magmático controlado estructuralmente que se propone en este trabajo.

En los últimos cinco años, la Agencia Nacional de Hidrocarburos de Colombia (ANH), patrocinó la obtención de nuevos datos geocronológicos (Tabla 1) y geoquímicos (Tablas 2 y 3 ) del occidente colombiano. El objetivo de este trabajo es definir el marco tectónico en el cual ocurrió el evento magmático Paleoceno-Eoceno, basados en la relación del evento magmático con el control estructural, así como en la continuidad espacial y temporal de los cuerpos ígneos. El análisis de los datos nuevos y los recopilados (Tabla 4), incluyen dataciones $\mathrm{U} / \mathrm{Pb}$ en circón y análisis de elementos mayores y tierras raras (Tablas S1 y S2, en suplemento electrónico). A partir de esa información, aquí se propone un modelo alternativo de evolución tectónica del occidente colombiano durante el lapso $60 \mathrm{Ma}$ a $26 \mathrm{Ma}$.

\section{MARCO TECTÓNICO REGIONAL}

La evolución tectónica del centro y sur del segmento Andino durante el Cretácico Tardío y Cenozoico, fue caracterizada por la evolución de un sistema de subducción entre las placas Farallón y Sudamérica, que dio lugar a magmatismo en la placa subducente y continuos apilamientos tectónicos bajo un régimen transpresivocompresivo (McCourt et al., 1984; Aspden et al., 1987; Grosser, 1989; Duque-Caro, 1990; Estrada, 1995; Cediel et al., 2003; Pindell y Kennan, 2009; López, 2009; Lissina, 2005; Ramos, 2009; Whattam y Stern, 2014; Montes et al., 2012; Bayona et al., 2012; Pindell et al., 2005; Villagómez et al., 2011; Cardona et al., 2018; Montes et al., 2019; Zapata-García y Rodríguez-García, 2020) (Figura 1). Presentamos a continuación una breve perspectiva histórica de cómo han evolucionado las ideas sobre la tectónica de la margen pacífica colombiana para el Cretácico Tardío y Cenozoico.

Barrero (1979) sugiere para el Cretácico Tardío el predominio de las fases extensionales en el borde de la placa Sudamericana, bajo un ambiente de subducción. Dicho régimen tectónico dio lugar a la formación de fosas y pilares tectónicos y al ascenso fisural de magmas toleíticos bajos en potasio, actualmente catalogados como tipo meseta oceánica. Este vulcanismo basáltico es parte del Grupo Diabásico y hace parte del basamento de las cuencas "onshore" Tumaco y Chocó (Figura 1a) de la margen pacífica colombiana (Barrero, 1979).

En contraposición a las ideas de Barrero (1979), autores como McCourt et al. (1984) y Grosser (1989) proponen varias acreciones sucesivas de terrenos alóctonos con basamento oceánico y continental en el borde occidental colombiano, en un sistema de subducción. Los terrenos acrecionados al continente durante el Cretácico Tardío fueron continentales, mientras que los terrenos oceánicos fueron acrecionados durante el Mioceno medio (Etayo-Serna et al., 1983; Restrepo y Toussaint, 2020). Durante el Mioceno Tardío fue acrecionado el Bloque Panamá-Chocó, reconocido por Duque-Caro (1990) como un terreno exótico que no tiene ninguna afinidad genética con Suramérica (Figura 1b). Presenta anomalías gravimétricas positivas asociadas a cuerpos ígneos de origen oceánico y está compuesto por tres elementos litotectónicos, el Arco de Dabeiba y Baudó, la Cuenca Atrato-Chucunaque y la Zona de Deformación de Istmina (DuqueCaro, 1990). El límite sur del Bloque Panamá-Chocó es la Zona de Deformación de Istmina y al oriente podría ser la zona de falla de Uramita (Figura 1a). Con respecto a la falla de Uramita Duque-Caro (1990) comenta: "la naturaleza de esta zona de falla es aún muy difícil de precisar debido a la falta de datos estructurales más detallados. Sin embargo, la ocurrencia exótica de los foraminíferos paleocenos, originalmente provenientes del norte, junto con la naturaleza tectónica del límite oriental del Arco de Dabeiba, sugieren que el lineamiento de Uramita es el contacto entre el Arco de Dabeiba de afinidad centroamericana y el noroccidente suramericano". Estrada (1995) se une a la interpretación de Duque-Caro (1990) de terrenos alóctonos acrecionados al NW de Colombia durante el Mioceno (terrenos Gorgona, Chocó y Cordillera Occidental), basando su interpretación de análisis paleomagnéticos realizados en basaltos almhoadillados de origen oceánico y en plutones del Terciario tardío (6-10 Ma). 


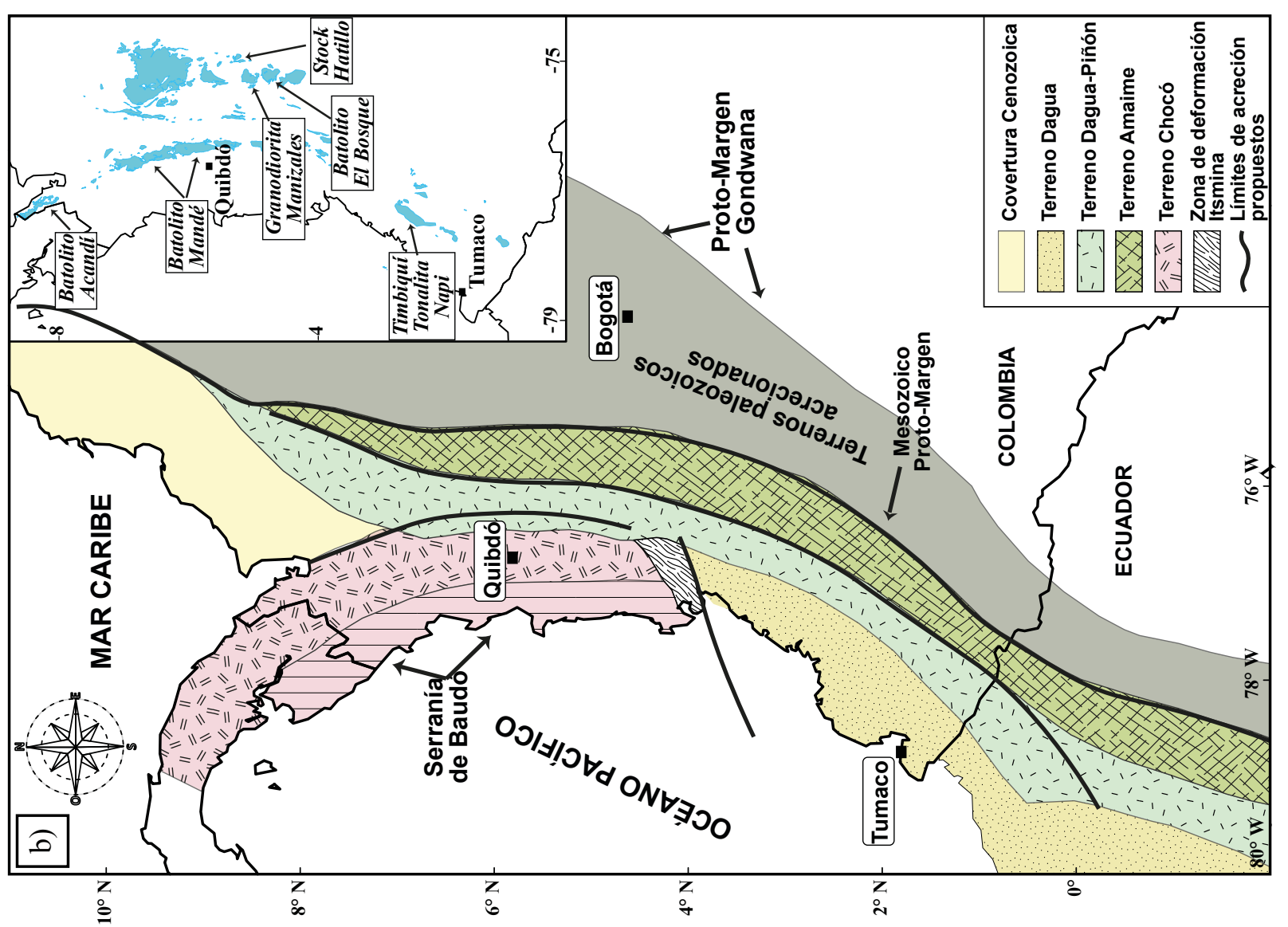

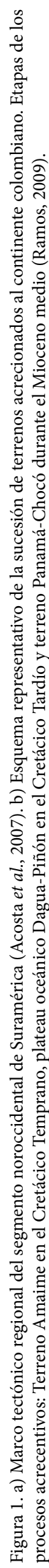

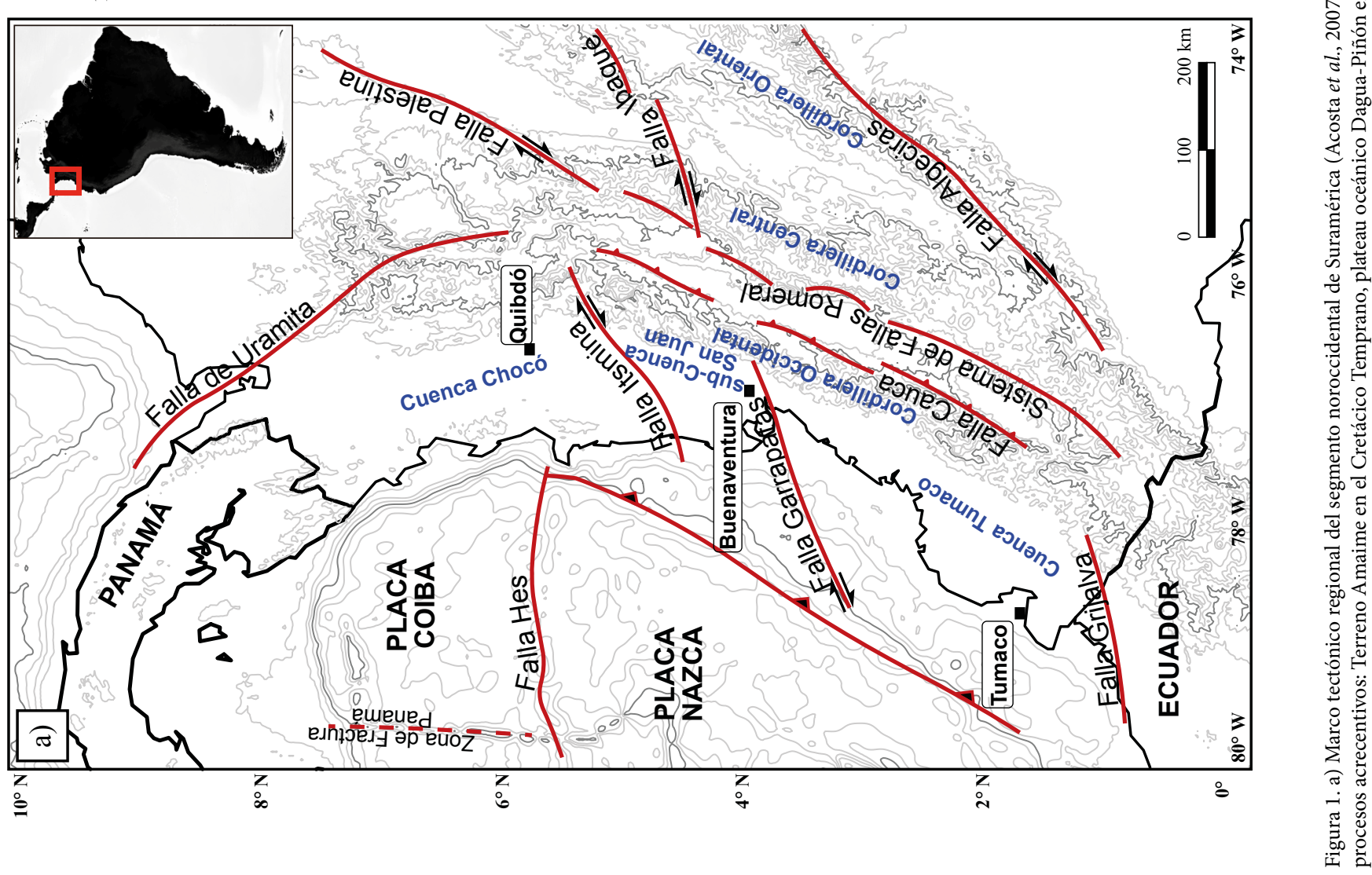


Tabla 1. Edades isotópicas $\mathrm{U} / \mathrm{Pb}$ en circones de unidades litológicas de la Cordillera Occidental. Desviación media ponderada al cuadrado (MSWD).

\begin{tabular}{lllllll}
\hline Muestra & Longitud & Latitud & Unidad & $\begin{array}{c}\text { Edad (Ma) } \\
{ }^{206} \mathrm{~Pb} /{ }^{238} \mathrm{U}\end{array}$ & MSWD Tipo de Roca & \\
\hline $1246-1$ & -77.17032 & 8.32407 & Batolito de Acandí & $46.27 \pm 0.28$ & 1.5 & Tonalitas - granodioritas \\
$1246-4$ & -77.18290 & 8.31515 & Batolito de Acandí & $46.07 \pm 0.49$ & 3.1 & Tonalitas - granodioritas \\
$40-006-C P C$ & -76.34890 & 6.34199 & Batolito de Mandé & $50.5 \pm 4.1$ & 2.2 & Granodiorita - tonalita \\
$90-005-C P C$ & -76.35251 & 6.33448 & Batolito de Mandé & $52.9 \pm 1.5$ & 3.8 & Tonalita horbléndica \\
$40-007-C P C$ & -76.33392 & 6.36684 & Mandé-Santa Cecilia la Equis & $59.2 \pm 1.6$ & 2.0 & Tonalita porfirítica \\
\hline
\end{tabular}

Un trabajo relevante es el de Cediel et al. (2003), quienes presentan una síntesis de los trabajos que tratan sobre el margen pacífico colombiano, y que fueron realizados en las tres décadas anteriores a la publicación de su trabajo. De acuerdo con esos autores, la porción noroeste de la margen colombiana consiste en el Terreno Chocó, el cual contiene los terrenos de Cañas Gordas y Baudó, de edad cretácica, que posteriormente fueron acrecionados durante el Mioceno medio al segmento norte de la margen pacífica colombiana. Durante el Paleoceno-Eoceno en el Terreno Chocó y el terreno Cañas Gordas, se instalaron los intrusivos de Mandé y Acandí (Figura 1b) que tienen una afinidad oceánica, determinada a partir análisis geoquímicos. Estos cuerpos intrusivos no presentan relación con los batolitos emplazados en la Cordillera Central (Batolito el Bosque, Granodiorita Manizales y stock El Hatillo), debido a que dichos batolitos de la Cordillera Central tienen una afinidad autóctona. La acreción del terreno Cañas Gordas comenzó en el Mioceno como una colisión altamente destructiva con una cinemática moderadamente destral oblicua. Dos evidencias reportadas por Cediel et al. (2003) sobre la colisión destructiva son: 1. "Las estructuras asociadas con la colisión del terreno Cañas Gordas deforman el terreno Gorgona y el sistema de fallas Buenaventura, que estaban en su lugar a principios del Mioceno" 2. "La falla de Garrapatas parece controlar la sedimentación del Mioceno al Holoceno a lo largo de la margen del Pacífico". Así entonces, con esas evidencias y basado en la sugerencia dada por Duque-Caro (1990), los autores se adhieren al modelo de acreción del Bloque Chocó en el Mioceno.

Más recientemente Montes et al. (2012) definieron varios eventos magmáticos: (1) arco magmático Cretácico Tardío hasta el Eoceno medio (58-39 Ma), asociado a subducción, (2) gap magmático desde el Eoceno tardío hasta el Oligoceno tardío (38-28 Ma), (3) reinicio del magmatismo a los $25 \mathrm{Ma}$. Estos eventos fueron parte de un proceso de colisión y acreción del borde de la Placa Caribe al continente, durante el Mioceno (Montes et al., 2019). Así mismo, Cardona et al. (2018) definieron dos arcos magmáticos Paleocénicos, no-cogenéticos, de afinidades continental y oceánica. Uno de ellos está ubicado en la parte norte de la Cordillera Occidental de Colombia y en Panamá, formado de rocas plutónicas y volcánicas con edades de 60-45 Ma, y su origen fue asociado a un ambiente tectónico tipo Aleutiano. El segundo es el arco de Timbiquí, que probablemente hizo parte de un terreno alóctono acrecionado durante el Eoceno tardío-Oligoceno (Echeverri et al., 2015; Cardona et al., 2018). Actualmente, desde el punto de vista geomorfológico, los cuerpos intrusivos están ubicados en la topografía positiva al occidente de Colombia, que es denominada la Cordillera

Tabla 2. Resultados analíticos de óxidos mayores (\% Wt) del Stock de Timbiquí. Pérdida por ignición (LOI).

\begin{tabular}{|c|c|c|c|c|c|c|c|c|c|c|c|c|}
\hline Muestra & $\mathrm{SiO}_{2}$ & $\mathrm{TiO}_{2}$ & $\mathrm{Al}_{2} \mathrm{O}_{3}$ & $\left(\mathrm{Fe}_{2} \mathrm{O}_{3}\right)_{\mathrm{t}}$ & MnO & $\mathrm{MgO}$ & $\mathrm{CaO}$ & $\mathrm{Na}_{2} \mathrm{O}$ & $\mathrm{K}_{2} \mathrm{O}$ & $\mathbf{P}_{2} \mathbf{O}_{5}$ & LOI & Total \\
\hline APO-0056-LG & 55.47 & 0.6 & 17.67 & 7.81 & 0.15 & 4.26 & 8.72 & 3.04 & 0.11 & 0.09 & -- & 97.92 \\
\hline APO-0049-LG & 60.64 & 0.47 & 17.55 & 5.95 & 0.15 & 2.93 & 5.77 & 3.52 & 0.92 & 0.15 & -- & 98.05 \\
\hline APO-0107-D & 56.93 & 0.52 & 17.65 & 7.79 & 0.17 & 3.39 & 8.07 & 2.96 & 0.23 & 0.13 & -- & 97.84 \\
\hline APO-0091-P & 59.25 & 0.45 & 18.14 & 5.51 & 0.16 & 1.79 & 5.33 & 3.52 & 3.31 & 0.26 & -- & 97.72 \\
\hline CAQ-0416-P & 42.9 & 0.84 & 18.69 & 13.04 & 0.21 & 5.96 & 12.99 & 1.97 & 0.78 & 0.56 & -- & 97.94 \\
\hline MZD-0010-L & 53.09 & 0.58 & 18.95 & 7.93 & 0.21 & 2.94 & 8.48 & 3.46 & 2.31 & 0.43 & -- & 98.38 \\
\hline MZD-0022-R & 64.43 & 0.28 & 16.79 & 3.64 & 0.08 & 1.05 & 4.99 & 4.12 & 1.43 & 0.13 & -- & 96.94 \\
\hline CLM-0447-L & 52.78 & 0.7 & 18.09 & 8.72 & 0.16 & 4.98 & 9.13 & 2.98 & 0.36 & 0.12 & -- & 98.02 \\
\hline CDG-0255-P & 54.16 & 0.85 & 16.99 & 7.98 & 0.13 & 5.07 & 10.13 & 2.51 & 0.04 & 0.12 & -- & 97.98 \\
\hline CDG-0256-P & 52.19 & 0.63 & 16.95 & 7.8 & 0.15 & 6.87 & 12.44 & 1.57 & 0.04 & 0.06 & -- & 98.7 \\
\hline MZD-0014-R & 41.08 & 0.98 & 18.79 & 14.08 & 0.24 & 6.13 & 14.07 & 1.38 & 0.73 & 0.89 & 1.3 & 99.67 \\
\hline APO-0044-LG & 47.49 & 0.12 & 24.58 & 6.5 & 0.15 & 6.12 & 12.72 & 1.06 & 0.05 & 0.01 & 1 & 99.8 \\
\hline CAQ-0346-P & 46.34 & 0.56 & 15.69 & 8.28 & 0.06 & 7.38 & 7.28 & 1.03 & 1.28 & 0.05 & 11.8 & 99.75 \\
\hline CLM-0377-P & 53.19 & 0.66 & 17.7 & 9.03 & 0.27 & 5.14 & 7.61 & 2.28 & 0.08 & 0.09 & 3.8 & 99.85 \\
\hline CLM-0388-P & 52.94 & 0.66 & 19.96 & 4.68 & 0.1 & 5.15 & 8.32 & 4.8 & 0.45 & 0.12 & 2.6 & 99.78 \\
\hline CLM-0445-P & 64.47 & 0.37 & 16.68 & 4.89 & 0.13 & 2.31 & 5.35 & 3.14 & 0.28 & 0.09 & 2.2 & 99.91 \\
\hline APO-0036-P & 58.14 & 0.35 & 18.73 & 3.33 & 0.11 & 1.54 & 6.34 & 3.62 & 1.3 & 0.19 & 6.2 & 99.85 \\
\hline APO-0054-LG & 61.65 & 0.52 & 16.87 & 6.57 & 0.15 & 2.7 & 4.91 & 3.86 & 0.18 & 0.13 & 2.3 & 99.84 \\
\hline APO-0065-LT & 54.39 & 0.55 & 17.44 & 7.91 & 0.19 & 3.22 & 7.25 & 2.81 & 1.77 & 0.25 & 4 & 99.78 \\
\hline APO-0069-LT & 58.44 & 0.48 & 16.85 & 6.93 & 0.21 & 2.8 & 6.58 & 3.48 & 0.9 & 0.16 & 3 & 99.83 \\
\hline CLM-0436-L & 65.75 & 0.34 & 16.19 & 4.4 & 0.19 & 2.05 & 3.87 & 3.98 & 0.62 & 0.12 & 2.4 & 99.91 \\
\hline CLM-0480-P & 58.06 & 0.75 & 17.12 & 8.03 & 0.09 & 3.25 & 1.2 & 1.46 & 2.18 & 0.13 & 7.5 & 99.77 \\
\hline
\end{tabular}


Tabla 3. Análisis geoquímicos de elementos traza y tierras raras en ppm, en muestras del Stock de Timbiquí.

\begin{tabular}{|c|c|c|c|c|c|c|c|c|c|c|c|c|c|c|c|c|c|c|}
\hline Muestra & $\mathrm{Ba}$ & $\mathrm{Ce}$ & Co & Cs & $\mathrm{Cu}$ & Dy & Er & Eu & Ga & Gd & Hf & Ho & La & Lu & $\mathrm{Nb}$ & Nd & $\mathbf{N i}$ & $\mathbf{P b}$ \\
\hline APO-0049-LG & 348.0 & 10.6 & 10.3 & 1.0 & 37.9 & 2.2 & 1.4 & 0.6 & 14.9 & 1.9 & 1.3 & 0.5 & 5.0 & 0.2 & 1.6 & 5.7 & 3.7 & 0.6 \\
\hline CAQ-0416-P & 162.0 & 15.2 & 32.1 & 0.3 & 237.4 & 3.1 & 1.5 & 1.3 & 16.5 & 3.6 & 0.5 & 0.6 & 6.9 & 0.2 & 0.7 & 14.8 & 8.4 & 0.6 \\
\hline MZD-0010-L & 510.0 & 19.4 & 14.4 & 0.2 & 27.1 & 2.7 & 1.9 & 1.0 & 16.3 & 3.4 & 1.6 & 0.6 & 9.5 & 0.3 & 1.7 & 12.2 & 0.3 & 0.2 \\
\hline MZD-0022-R & 358.0 & 18.3 & 3.8 & $<0.1$ & 9.4 & 2.3 & 1.4 & 0.7 & 12.9 & 2.1 & 2.3 & 0.5 & 10.8 & 0.3 & 2.5 & 10.9 & 0.5 & 0.2 \\
\hline CAQ-0346-P & 41.0 & 5.5 & 24.7 & 0.4 & 50.5 & 1.9 & 1.3 & 0.5 & 12.8 & 1.6 & 0.9 & 0.4 & 1.8 & 0.2 & 0.3 & 4.8 & 22.9 & 0.7 \\
\hline CLM-0377-P & 25.0 & 5.1 & 22.3 & $<0.1$ & 137.9 & 3.0 & 2.0 & 0.6 & 14.0 & 2.3 & 1.1 & 0.7 & 1.9 & 0.3 & 0.3 & 5.2 & 5.7 & 1.0 \\
\hline CLM-0388-P & 113.0 & 5.5 & 16.1 & 0.1 & 14.8 & 2.6 & 1.5 & 0.6 & 13.3 & 1.9 & 1.0 & 0.5 & 2.4 & 0.3 & 0.3 & 4.3 & 74.1 & 0.9 \\
\hline CLM-0445-P & 98.0 & 7.4 & 7.5 & 0.1 & 22.4 & 2.0 & 1.3 & 0.5 & 12.9 & 1.7 & 1.5 & 0.5 & 3.5 & 0.3 & 0.6 & 5.7 & 4.7 & 0.7 \\
\hline APO-0036-P & 334.0 & 24.7 & 4.7 & 0.5 & 17.2 & 2.1 & 1.2 & 0.9 & 17.1 & 2.4 & 1.9 & 0.4 & 13.1 & 0.2 & 3.4 & 15.7 & 0.9 & 4.5 \\
\hline APO-0054-LG & 215.0 & 11.3 & 12.4 & $<0.1$ & 60.8 & 2.7 & 1.7 & 0.7 & 14.0 & 2.5 & 1.6 & 0.6 & 5.0 & 0.3 & 0.8 & 7.6 & 5.5 & 0.4 \\
\hline CLM-0480-P & 427.0 & 27.3 & 19.1 & 2.3 & 110.5 & 2.7 & 1.7 & 0.9 & 16.4 & 3.0 & 3.1 & 0.6 & 13.6 & 0.3 & 5.1 & 13.2 & 44.4 & 6.7 \\
\hline CAQ-0415R & 402.0 & 20.4 & -- & 0.4 & -- & 2.7 & 1.5 & 1.4 & -- & 3.2 & -- & 0.6 & 10.4 & 0.3 & 1.5 & 13.7 & -- & 0.4 \\
\hline CAQ-0420R & 166.0 & 15.7 & -- & 0.7 & -- & 3.2 & 1.9 & 0.9 & -- & 3.3 & -- & 0.7 & 6.6 & 0.3 & 1.9 & 11.7 & -- & 0.7 \\
\hline CLM-0447-L & 91.0 & 9.4 & 22.5 & $<0.1$ & 81.0 & 2.8 & 1.8 & 0.7 & 14.0 & 2.4 & 1.6 & 0.6 & 3.9 & 0.3 & 0.8 & 7.3 & 6.3 & 0.4 \\
\hline APO-0091-P & 656.0 & 26.3 & 9.1 & 0.4 & 52.9 & 2.8 & 1.9 & 1.1 & 16.7 & 3.1 & 2.6 & 0.6 & 14.4 & 0.3 & 3.6 & 13.9 & 1.3 & 0.2 \\
\hline CDG-0255-P & 27.0 & 9.0 & 17.6 & 0.1 & 1.7 & 3.2 & 1.9 & 0.9 & 14.1 & 2.7 & 1.8 & 0.7 & 3.2 & 0.3 & 1.1 & 7.4 & 4.2 & 0.2 \\
\hline CDG-0256-P & 20.0 & 5.4 & 25.8 & 0.1 & 3.4 & 2.1 & 1.5 & 0.6 & 12.8 & 1.8 & 1.0 & 0.5 & 2.2 & 0.2 & 0.7 & 5.0 & 4.2 & 0.1 \\
\hline
\end{tabular}

Occidental. Desde el punto de vista genético y geológico, estos cuerpos intrusivos han sido atribuidos como parte del Bloque Panamá-Chocó. Por lo tanto, estos cuerpos son parte de la Cordillera Occidental y, según los modelos propuestos en las últimas cuatro décadas, también del Bloque Panamá-Chocó.

Zapata-García y Rodríguez-García (2020) muestran evidencias de la continuidad del arco magmático Paleoceno-Eoceno. Adicionan al Bloque Panamá-Chocó la Formación Timbiquí y la Tonalita de Napi (Figura 1b), ubicadas al sur de la Cordillera Occidental de Colombia. A partir de análisis geoquímicos, geología de campo, geocronología y petrografía, muestran que rocas volcánicas y plutónicas que constituyen el Arco Panamá-Chocó norte (Complejo Santa Cecilia-La Equis y Batolito de Acandí), central (Complejo Santa Cecilia-La Equis y Batolito de Mandé) y sur (Formación Timbiquí y Tonalita de Napi) son similares en litología, geoquímica, composición y edad (Eoceno).

El magmatismo Paleoceno temprano en la Cordillera Central ha sido atribuido a procesos relacionados con la flotabilidad del slab de subducción de la placa Caribe (Bayona et al., 2012; Cardona et al., 2018). Análisis geocronológicos de $\mathrm{U} / \mathrm{Pb}$ en cuerpos intrusivos y circones detríticos, indican el aporte de sedimentos de edad Paleoceno para las cuencas intramontanas. Se ha propuesto que la parada repentina del magmatismo a principios del Eoceno medio puede estar relacionado con la dificultad de la subducción de un grueso plateau oceánico, dada por el movimiento transcurrente entre la Placa Sudamericana y Caribe (Aspden et al., 1987; Bayona et al., 2012; Pindell et al., 2005; Villagómez et al., 2011; Cardona et al., 2018).

\section{METODOLOGÍA}

Se realizaron cinco dataciones isotópicas usando el método U/ $\mathrm{Pb}$ en circones de cuerpos ígneos (Figura 2, Tabla S3 del suple- mento electrónico), en cuerpos ígneos localizados a lo largo de la CO. Se utilizó la técnica de análisis espectrométrico de relaciones isotópicas en granos individuales con ablación por punto ("spot analysis") utilizado un sistema de laser acoplado a un espectrómetro de masas (Laser Ablation-Multicollector-Inductively Coupled Plasma-Mass Spectrometry o LA-ICP-MS) (Tabla 1, Figura 3). Se realizaron también análisis geoquímicos en roca entera, incluyendo veintidós (22) muestras para elementos mayores y veinticuatro (24) para elementos traza. Los análisis de elementos mayores y traza se llevaron a cabo mediante el método ICP-MS (Inductively Coupled Plasma-mass spectrometry) en ACMELABS (Analytical Laboratories, Vancouver, Canadá). La precisión lograda con este procedimiento es de \pm 2 ppm y \pm 5 ppm para una concentración analítica entre 50 y 5 ppm, respectivamente. Para cada muestra, fueron tomados $0.2 \mathrm{~g}$ y se mezclaron con $1.5 \mathrm{~g}$ de flujo de $\mathrm{LiBO}_{2}$. Fueron calentados a $1050{ }^{\circ} \mathrm{C}$ durante $15 \mathrm{~min}$. Posteriormente se disolvieron en $\mathrm{HNO}_{3}$ al $5 \%$. Patrones de calibración y blancos de reactivo fueron añadidos, y las soluciones se aspiraron en un espectrómetro de emisión ICP. Los límites de detección de los elementos mayores varían entre $0.001 \%$ y $0.004 \%$, y los valores de los oligoelementos son de aproximadamente $0.1 \mathrm{ppm}$. Los elementos de tierras raras tienen límites de detección entre 0.01 y 0.05 ppm. Estos resultados se presentan libres de $\mathrm{H}_{2} \mathrm{O}^{+}, \mathrm{H}_{2} \mathrm{O}, \mathrm{CO}_{2}$ y normalizados a $100 \%$. Las muestras con pérdida por ignición mayor a $3.5 \%$ fueron descartadas de los análisis debido a que estas pérdidas se consideran como un índice de alteración de la roca. Los resultados de los análisis geoquímicos se manejaron y procesaron utilizando el software GCD Kit 4.1 (Janousek et al., 2006) (Tablas 2 y 3 ).

Se recopilaron treinta y cinco (35) análisis geocronológicos $\mathrm{U} / \mathrm{Pb}$ (Tabla 4), sesenta y siete (67) análisis de elementos mayores y cuarenta y cuatro (44) de elementos traza (Tablas S1 y S2), disponibles a partir de investigaciones publicadas y trabajos e informes cartográficos del Servicio Geológico Colombiano y la ANH. 
Tabla 4. Edades isotópicas compiladas de la literatura del evento magmático Paleoceno - Eoceno, Cordilleras Occidental y Central de Colombia.

\begin{tabular}{|c|c|c|c|c|c|c|c|}
\hline Muestra & Longitud & Latitud & Nombre Unidad & Edad (Ma) & Método & Tipo de roca & Referencia \\
\hline DV64 & -74.95536 & 5.98236 & Batolito Antioqueño & $58.9 \pm 9.6$ & $\mathrm{U} / \mathrm{Pb} \mathrm{Cir}$ & Granodiorita & Villagómez et al., 2011 \\
\hline DV148 & -75.38492 & 6.42119 & Batolito Antioqueño & $53 \pm 4.8$ & $\mathrm{U} / \mathrm{Pb} \mathrm{Cir}$ & Granodiorita & Villagómez et al., 2011 \\
\hline GR-II-1 & -74.91191 & 6.51065 & Batolito Antioqueño & $59.2 \pm 1.2$ & $\mathrm{U} / \mathrm{Pb} \mathrm{Cir}$ & Tonalita biotítica & Leal Mejía, 2011 \\
\hline G-29 & -74.92009 & 6.51047 & Batolito Antioqueño & $60.7 \pm 1$ & $\mathrm{U} / \mathrm{Pb}$ Cir & Tonalita biotítica & Leal Mejía, 2011 \\
\hline WR-216 & -75.203548 & 5.66734 & Batolito de Sonsón & $55.8 \pm 0.9$ & $\mathrm{U} / \mathrm{Pb} \mathrm{Cir}$ & Leucogranito & Leal Mejía, 2011 \\
\hline WR-218 & -75.19388 & 5.65845 & Batolito de Sonsón & $60 \pm 0.9$ & $\mathrm{U} / \mathrm{Pb} \mathrm{Cir}$ & Leucogranito & Leal Mejía, 2011 \\
\hline 07DV156 & -75.300009 & 5.75369 & Batolito de Sonsón & $59 \pm 0.5$ & $\mathrm{U} / \mathrm{Pb} \mathrm{Cir}$ & Granito & Cochrane, 2013 \\
\hline BSS-5 & -75.298104 & 5.75591 & Batolito de Sonsón & $60.7 \pm 1.4$ & $\mathrm{U} / \mathrm{Pb} \mathrm{Cir}$ & Cuarzo diorita & Ordóñez-Carmona et al., 2011 \\
\hline 69 & -75.32995 & 5.95556 & Stock de La Unión & $59 \pm 12$ & $\mathrm{U} / \mathrm{Pb} \mathrm{Cir}$ & Tonalita & Restrepo et al., 1991 \\
\hline $\mathrm{CH} 10$ & -74.98982 & 5.14893 & Stock el Hatillo & $55.0 \pm 0.7$ & $\mathrm{U} / \mathrm{Pb} \mathrm{Cir}$ & -- & Bustamante et al., 2016 \\
\hline GCC-11 & -74.97359 & 5.17379 & Stock el Hatillo & $54.6 \pm 0.7$ & $\mathrm{U} / \mathrm{Pb} \mathrm{Cir}$ & Cuarzodiorita & Bayona et al., 2012 \\
\hline CB1 & -75.14055 & 4.73725 & Batolito El Bosque & $55.1 \pm 2.1$ & $\mathrm{U} / \mathrm{Pb} \mathrm{Cir}$ & -- & Bustamante et al., 2016 \\
\hline DV163 & -74.97822 & 5.17877 & Plutón de El Hatillo & $55.3 \pm 5.4$ & $\mathrm{U} / \mathrm{Pb}$ Cir & Granito & Villagómez et al., 2011 \\
\hline DV04 & -74.97539 & 4.78339 & Batolito de Ibagué & $59.2 \pm 20.2$ & $\mathrm{U} / \mathrm{Pb} \mathrm{Cir}$ & Gabrodiorita & Villagómez et al., 2011 \\
\hline DV05 & -75.26814 & 4.40769 & Batolito de Ibagué & $59.8 \pm 16.8$ & $\mathrm{U} / \mathrm{Pb} \mathrm{Cir}$ & Granodiorita & Villagómez et al., 2011 \\
\hline ER-1 & -75.09912 & 6.48492 & Intrusivo pórfido El Rayo & $59.9 \pm 0.9$ & $\mathrm{U} / \mathrm{Pb} \mathrm{Cir}$ & Pórfido aurífero & Leal Mejía, 2011 \\
\hline SG-002 & -75.85156 & 6.59481 & Batolito de Sabanalarga & $50.28 \pm 16.6$ & $\mathrm{U} / \mathrm{Pb} \mathrm{Cir}$ & -- & ANH-UCaldas, 2011 \\
\hline IGM-156841 & -75.41334 & 4.18053 & Pórfido de Río Manso & $58 \pm 10$ & $\mathrm{U} / \mathrm{Pb}$ Cir & Cuarzodiorita & Núñez, 1981 \\
\hline DV30 & -76.18066 & 3.90294 & Batolito de Buga & $44.8 \pm 8.4$ & $\mathrm{U} / \mathrm{Pb} \mathrm{Cir}$ & Tonalita & Villagómez et al., 2011 \\
\hline BSB1 & -76.15173 & 3.34943 & Batolito de Santa Barbara & $58.9 \pm 0.4$ & $\mathrm{U} / \mathrm{Pb} \mathrm{Cir}$ & Tonalita & Ordóñez-Carmona et al., 2011 \\
\hline BSB2 & -76.142849 & 3.37582 & Batolito de Santa Barbara & $58.4 \pm 0.8$ & $\mathrm{U} / \mathrm{Pb} \mathrm{Cir}$ & Tonalita & Ordóñez-Carmona et al., 2011 \\
\hline APO-0056-LG & -77.24070 & 2.74230 & Stock de Timbiquí & $46.8 \pm 0.7$ & $\mathrm{U} / \mathrm{Pb}$ Cir & Cuarzodiorita & ANH-GRP, 2014 \\
\hline WR-238 & -76.52136 & 6.726582 & Batolito de Mandé & $44.6 \pm 0.9$ & $\mathrm{U} / \mathrm{Pb}$ Cir & Pórfido tonalítico & Leal Mejía, 2011 \\
\hline WR-237 & -76.518721 & 6.723443 & Batolito de Mandé & $45.3 \pm 1.2$ & $\mathrm{U} / \mathrm{Pb} \mathrm{Cir}$ & Pórfido tonalítico & Leal Mejía, 2011 \\
\hline $\mathrm{XX}$ & -77.404248 & 8.524412 & Batolito de Acandí & 49.5 & $\mathrm{U} / \mathrm{Pb} \mathrm{Cir}$ & -- & Cardona et al., 2018 \\
\hline $\mathrm{X}$ & -77.592307 & 2.045487 & Stock de Timbiquí & 44.3 & $\mathrm{U} / \mathrm{Pb}$ Cir & -- & Cardona et al., 2018 \\
\hline
\end{tabular}

En total se realizó el análisis de cuarenta (40) datos geocronológicos $\mathrm{U} / \mathrm{Pb}$, ochenta y nueve (89) análisis de elementos mayores, y sesenta y ocho (68) de elementos traza. Todos los análisis de óxidos mayores se recalcularon al $100 \%$ teniendo en cuenta la pérdida por ignición (LOI).

\section{RESULTADOS}

Se integraron los nuevos análisis con los obtenidos de la bibliografía, para construir una base de datos geocronológicos y geoquímicos de rocas plutónicas/volcánicas del Paleoceno - Eoceno de la región estudiada. Los resultados son presentados resaltando los dominios en la CO y en la CC, enfocándose en la distribución, migración y tiempo de permanencia.

\section{Arco magmático en la Cordillera Occidental}

Los cinco (5) nuevos análisis realizados en esta investigación en conjunto con los reportados por ANH-GRP (2014), muestran que las edades de cristalización (U/Pb en circón) del arco magmático de Acandí y Mandé son muy cercanas a los intrusivos denominados Stock de Timbiquí, Diques Dioríticos, Intrusivo de Muchinca y Tonalita de Napi, ubicados en el sector sur de la CO (Figuras 3 y 4). Las composiciones de esos cuerpos intrusivos incluyen tonalita, cuarzodiorita, diorita, gabro con cuarzo y gabro horblendítico para la Tonalita de Napi. El Stock de Timbiquí está constituido por gabros, dioritas cuarzosas, andesitas, basaltos (tipo lavas almohadilladas). Los Diques Dioríticos están compuestos de diorita y cuarzodiorita. El Intrusivo de Muchinca son cuarzodioritas y microgabros. Estas rocas presentan series toleíticas a calco-alcalinas como se observa en la Figura 4. 

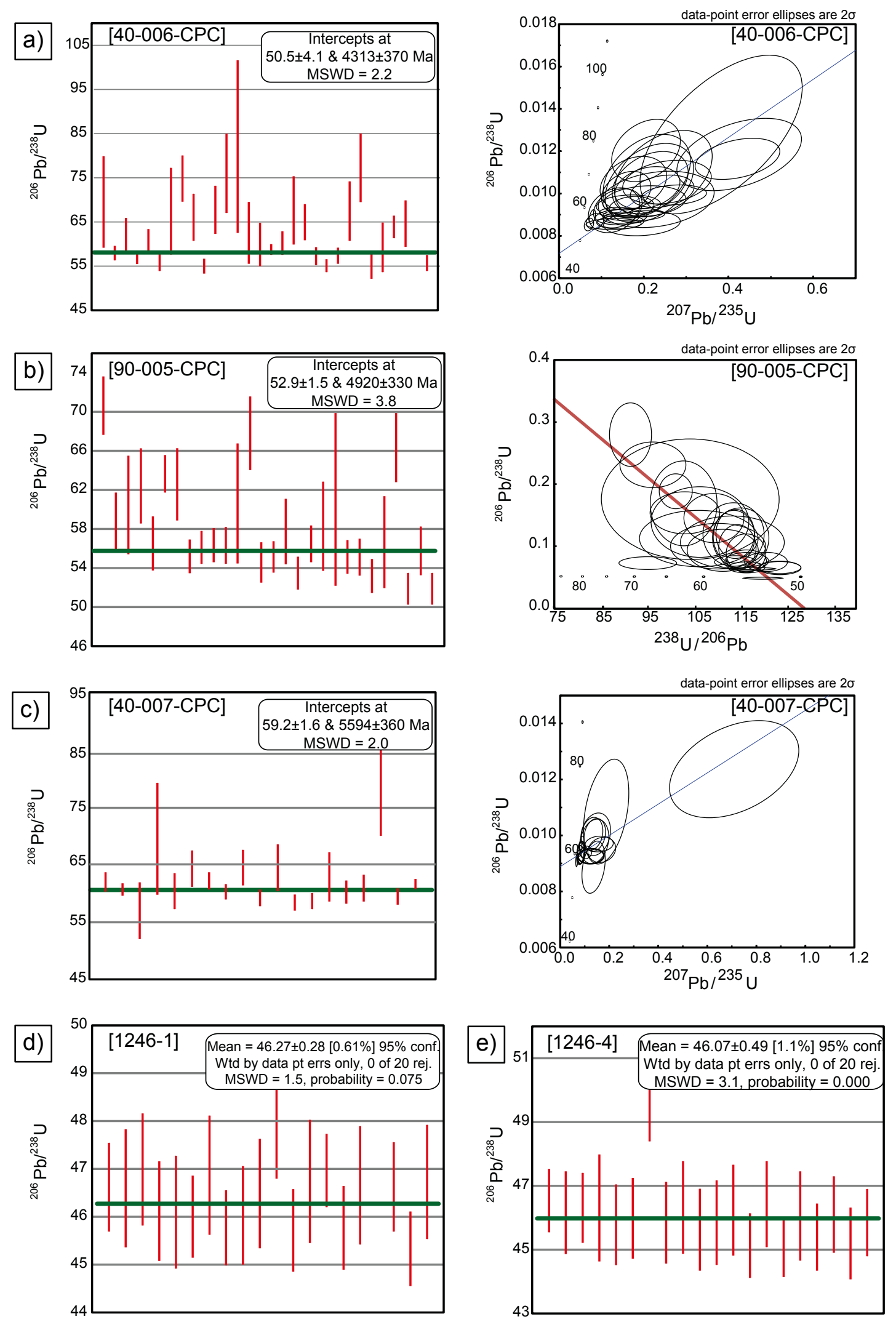

Figura 2. Geocronología U/Pb circón. a) y b) Batolito de Mandé, c) Mandé - Santa Cecilia la Equis, d) y e) Batolito de Acandí. [número de la muestra], desviación estándar media ponderada (MSWD). 


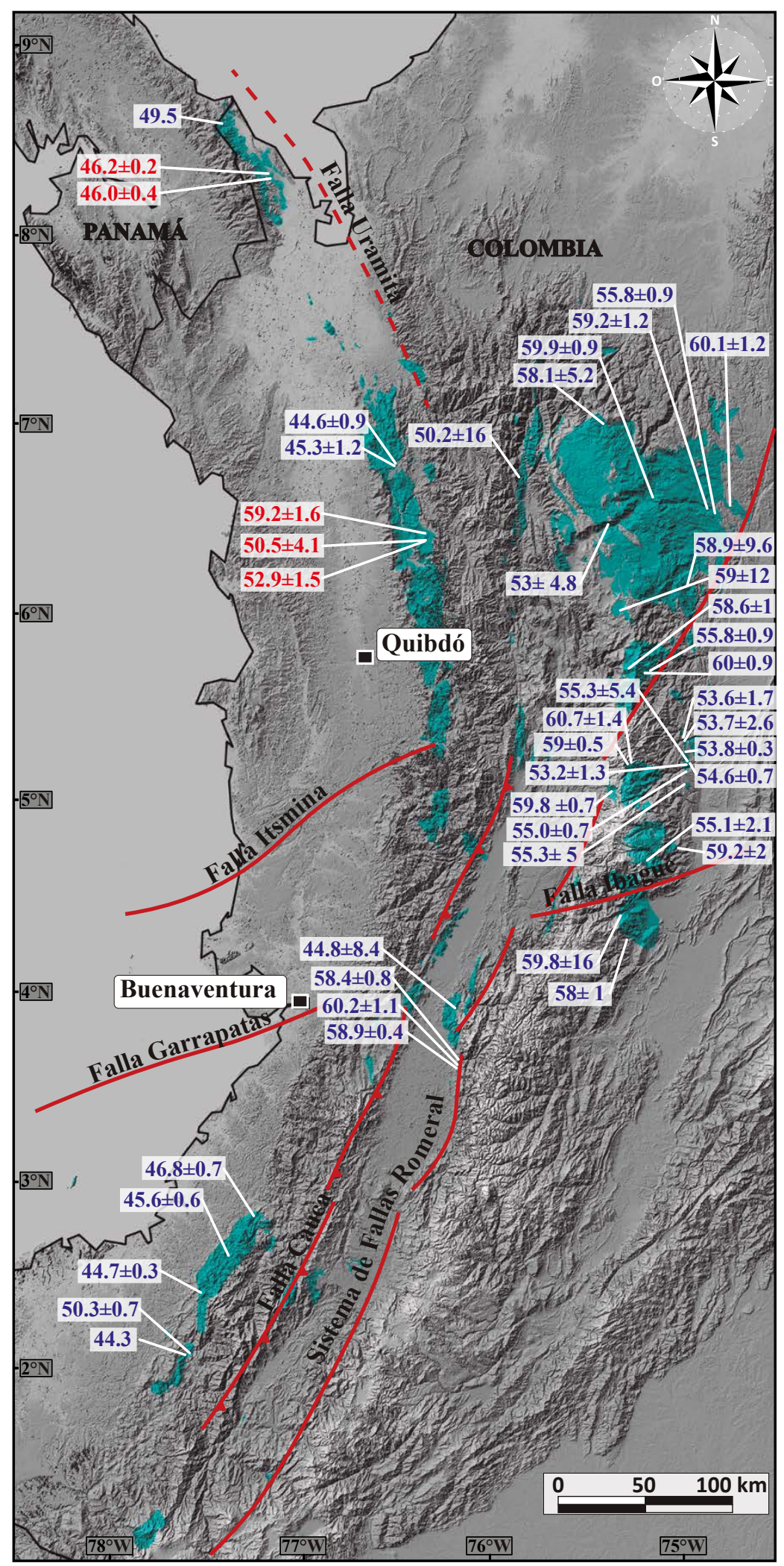

Figura 3. Distribución, geometría y tiempo de permanencia de las rocas de edad Paleoceno y Eoceno. Los análisis reportados son realizados a partir del método U/Pb en circón. En color rojo, se muestran las edades obtenidas en este trabajo. En color azul, los análisis U/Pb recopilados de la literatura (Núñez, 1981; Restrepo et al., 1991; Leal Mejía, 2011; ANH-UCaldas, 2011; Ordóñez-Carmona et al., 2011; Bayona et al., 2012; Villagómez y Spikings, 2013; ANH-GRP, 2013; Cochrane, 2013; Bustamante et al., 2016; Cardona et al., 2018). 
Considerando todas las edades ubicadas sobre la CO se observa que abarcan de $59.2 \mathrm{Ma}$ a 44.3 Ma. De norte (límites con Panamá) a sur (límites con Ecuador), se identifican las siguientes unidades: 1 . Batolito de Acandí, con edades de 49 Ma a 46 Ma. 2. Batolito de Mandé, con edades desde 59 Ma hasta $44 \mathrm{Ma}$. 3. El Stock de Timbiquí, Diques Dioríticos y el Intrusivo de Muchinca (entre $2^{\circ}$ a $3^{\circ}$ de latitud N) registran edades desde $50 \mathrm{Ma}$ hasta $44 \mathrm{Ma}$. (Figuras 2 y 3; Tabla 2). Composicionalmente, estos cuerpos están constituidos por variaciones de gabros hasta granitoides. En el Batolito de Mandé se observan facies más básicas hacia los bordes, diques basálticos y localmente con inclusiones máficas. (INGEOMINAS, 2002a, 2002b, 2010a, 2010b). En primera instancia, este evento magmático Paleoceno - Eoceno presenta una predominancia de magmatismo intermedio a básico, desde gabro hasta granodiorita (Figura 4b). Los diagramas de Peccerillo y Taylor (1976) y AFM muestran que el conjunto de análisis es una tendencia progresiva, de series toleíticas hacia series calcoalcalinas. (Figura 4c).

Los elementos mayores se graficaron en relación a la variación de la concentración del $\mathrm{SiO}_{2}$ (Figura 5a). Las concentraciones de $\mathrm{SiO}_{2}$ presentan valores menores a $65 \mathrm{wt} \%$, una tendencia a la disminución del $\mathrm{SiO}_{2}$ a mayores concentraciones en $\mathrm{CaO}, \mathrm{MgO}, \mathrm{Al}_{2} \mathrm{O}_{3}$ y FeOt; en contraste con el aumento de $\mathrm{SiO}_{2}$ para mayores concentraciones de $\mathrm{Na}_{2} \mathrm{O}$ y $\mathrm{K}_{2} \mathrm{O}$. En los elementos traza ( $\mathrm{Ba}, \mathrm{Ce}, \mathrm{La}, \mathrm{Rb}, \mathrm{Sr}, \mathrm{Zr}$ ) se observa una disminución progresiva y continua al decrecimiento de $\mathrm{SiO}_{2}$. El Ba tiene valores hasta de 600 ppm, el Ce y Rb hasta 30 ppm, el La hasta 15 ppm, el Sr hasta 500 ppm y el Zr hasta 100 ppm (Figura 5b).

Las edades y la composición química de los cuerpos magmáticos descritas en los párrafos anteriores sugieren fuertemente que hay continuidad del conjunto magmático, desde Acandí hasta el sector sur de la CO. Esta continuidad también fue propuesta por Zapata-García y Rodríguez-García (2020) desde un punto de vista geoquímico.

\section{Arco magmático en la Cordillera Central}

En la Cordillera Central (CC) las unidades plutónicas/volcánicas están concentradas principalmente a $4.5^{\circ}$ de latitud $\mathrm{N}$ y la mayor expresión está dada por el Batolito Antioqueño (Aspden et al., 1987). Las edades de cristalización (U/Pb en circón) de los cuerpos distribuidos en la CC están registradas desde el Cretácico Tardío hasta $53 \mathrm{Ma}$. Sin embargo, en este trabajo se considerarán sólo las dataciones en el rango Paleoceno - Eoceno (Figuras 3 y 4). De norte a sur, se tienen los intrusivos: 1. Batolito Antioqueño, Intrusivo pórfido el Rayo, Stock de Caracolí y Batolito de Sabanalarga, con edades que van de $60.1 \mathrm{Ma}$ a $50.2 \mathrm{Ma}$. 2. Stock de la Unión con una edad de $59 \mathrm{Ma}$. 3. Batolito de Sonsón que presenta edades de $60 \mathrm{Ma}$ a 55.8 Ma. 4. Stock El Hatillo varia de 53.8 Ma a 53.3 Ma. 5. Granodiorita de Manizales con edades de 60.7 Ma a 59 Ma. 6. Batolito El Bosque presenta edades al sur del cuerpo intrusivo de 55.1 Ma hasta 59.2 Ma. 7. Batolito de Buga de 44.8 Ma y otros cuerpos como el Batolito de Ibagué con edades de 59.8 a 58 $8 \mathrm{Ma}$ y Batolito de Santa Bárbara de $60.2 \mathrm{Ma}$ a 58.4 (Restrepo et al., 1991; Ordóñez-Carmona et al., 2011; Leal Mejía, 2011; ANH-UCaldas, 2011; Bayona et al., 2012; Villagómez y Spikings, 2013; Cochrane, 2013; Bustamante et al., 2016). Esos intrusivos están compuestos principalmente por granodiorita, tonalita, cuarzodiorita, cuarzomonsodiorita, granito y cuarzomonzonita. Localmente, algunos plutones contienen xenolitos de gneis y anfibolita, así como diques aplíticos (Núñez, 1981; INGEOMINAS, 2002a, 2002c, 2002d, 2002e). La composición en general es ácida y se clasifica en los campos de las granodioritas y granitos con afinidades calcoalcalinas (Figura 4).

La concentración del $\mathrm{SiO}_{2}$ está en rangos de 75 a 65 wt\%. La concentración de $\mathrm{Na}_{2} \mathrm{O}$ y $\mathrm{K}_{2} \mathrm{O}$ muestra un incremento con una tendencia positiva con respecto al $\mathrm{SiO}_{2}$. Por el contrario, el $\mathrm{Al}_{2} \mathrm{O}_{3}, \mathrm{FeO}, \mathrm{CaO}$ y $\mathrm{MgO}$, disminuyen su concentración con el incremento del $\mathrm{SiO}_{2}$. Los elementos traza para estos cuerpos muestran una correlación positi- va con respecto al contenido de $\mathrm{SiO}_{2}$. La concentración de $\mathrm{Ba}$ es de 600-1000 ppm, Ce de 30-70 pmm, La 15-40 ppm, Rb 20-60 ppm, Sr 500-700 ppm y Zr desde 100 hasta 150 pmm (Figura 5).

\section{DISCUSIÓN}

La discusión que se presenta aquí se restringe al registro del Paleoceno - Eoceno, los eventos magmáticos posteriores al Eoceno quedan fuera del objetivo de este trabajo.

\section{Tiempo de permanencia y migración del arco magmático}

El evento magmático en el rango Paleoceno - Eoceno está registrado por cuerpos intrusivos distribuidos en la CC que tienen edades de 60.7 Ma a 44.8 Ma (Figura 3 y 6), y representan un gran volumen de magma. Sobre la CO, en el arco de Panamá, hasta la parte centro-norte de la cordillera, representada por el Batolito de Acandí y el Batolito de Mandé, se tienen edades desde 59.2 Ma hasta 44.6 Ma y hacia el sur, en el sector de Tumaco, las edades de los cuerpos intrusivos varían de $50.3 \mathrm{Ma}$ a $44.3 \mathrm{Ma}$ (Figura 3 y 5 ).

Los cuerpos intrusivos con edades entre 60.7 Ma y 53 Ma se emplazaron simultáneamente en ambas cordilleras Occidental y Central (Figura 6). Sin embargo, las composiciones y geoquímica son diferentes, mientras en la CC predominan las granodioritas con series principalmente calco-alcalinas, en la CO predominan composiciones de granodiorita con variaciones composicionales hacia los bordes de los cuerpos a facies básicas, e inclusiones basálticas (Batolito de Mandé) y adicionalmente, numerosos diques andesíticos y basálticos. La geoquímica en los cuerpos intrusivos de la CO abarca desde series toleíticas hasta calcoalcalinas (Figuras 4 y 5 ).

A partir de los $53 \mathrm{Ma}$ y hasta los $44.3 \mathrm{Ma}$, la provincia magmática Paleoceno - Eoceno se concentra en la CO y en el flanco occidental de la CC, evidenciando la migración del arco magmático hacia el occidente (Figura 6).

Edades isotópicas muestran que la actividad magmática PaleocenoEoceno estuvo también presente en el Arco de Panamá. La actividad magmática inició alrededor de los 70 Ma y disminuyó después de los $38 \mathrm{Ma}$.

\section{Cinemática y control geométrico}

Para el análisis cinemático de la placa Farallón con respecto a la placa Sudamericana, considerando el lapso entre $60 \mathrm{Ma}$ y $44 \mathrm{Ma}$, se tomó como referencia una dirección de convergencia promedio de $65^{\circ}$ (Figura 7). Esta dirección es equivalente a la dirección de desplazamiento $\left(25^{\circ}\right.$ de azimut) de la placa Farallón con respecto al norte actual (Figura 8). Lo anterior se basa en los trabajos de Martinod et al. (2010), el cual incorpora estudios de cinemática realizados a lo largo de Suramérica, y el de Liu et al. (2008), que se refiere a la zona más cercana al límite norte de Colombia (Figura 7).

En términos de velocidad, la placa Farallón se desplazaba en promedio a $5 \mathrm{~cm} /$ año entre los 65 Ma y $30 \mathrm{Ma}$ (Pardo-Casas y Molnar, 1987; Daly, 1989; Somoza y Chidella, 2005). Considerando un azimut de $25^{\circ}$ para el vector de velocidad de desplazamiento de la placa Farallón con respecto a la margen de Suramérica (Figura 8), obtenemos que la componente ortogonal a la margen es de $2.1 \mathrm{~cm} /$ año, lo que representa el $10 \%$ de la velocidad total de convergencia. La componente paralela a la margen es de $4.5 \mathrm{~cm} /$ año, que corresponde al $90 \%$ de la velocidad total, como se observa en la Figura 8.

El ángulo de convergencia de la placa Farallón transfiere un esfuerzo compresivo principal máximo $\sigma 1$ horizontal en dirección paralela al vector de desplazamiento de la placa Farallón y un esfuerzo $\sigma 3$ horizontal en dirección ortogonal a $\sigma 1$, entonces se promovería el 
b)

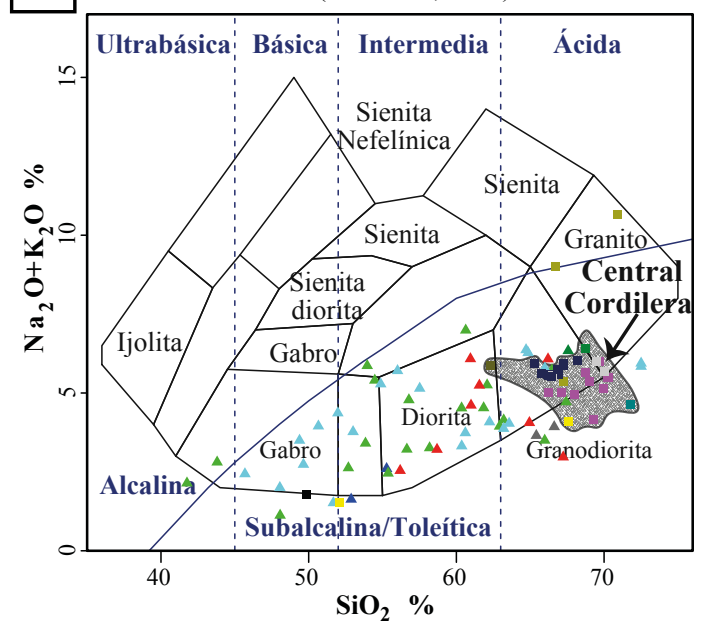

c) Diagramas de clasificación

$\mathrm{SiO}_{2}-\mathrm{K}_{2} \mathrm{O}$ plot (Peccerillo y Taylor, 1976)

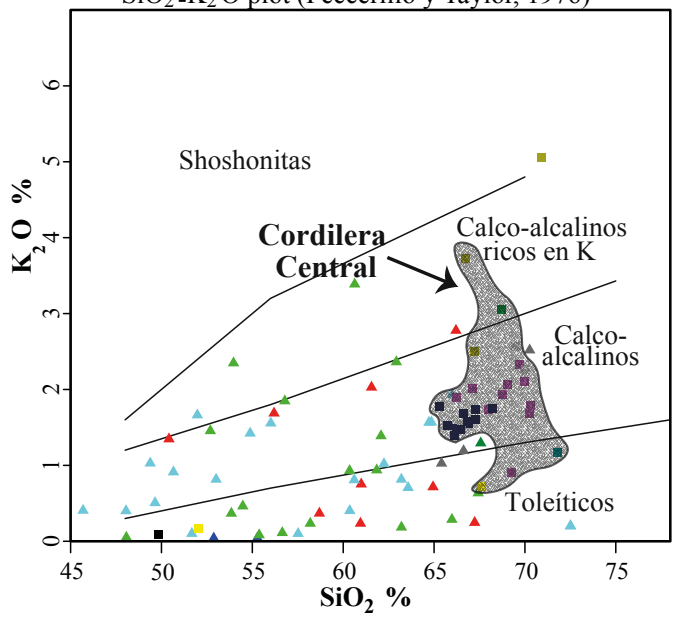

d)

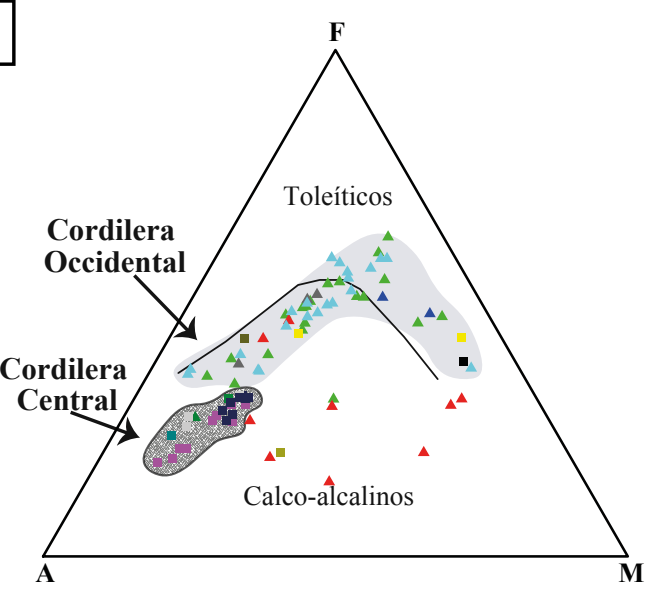

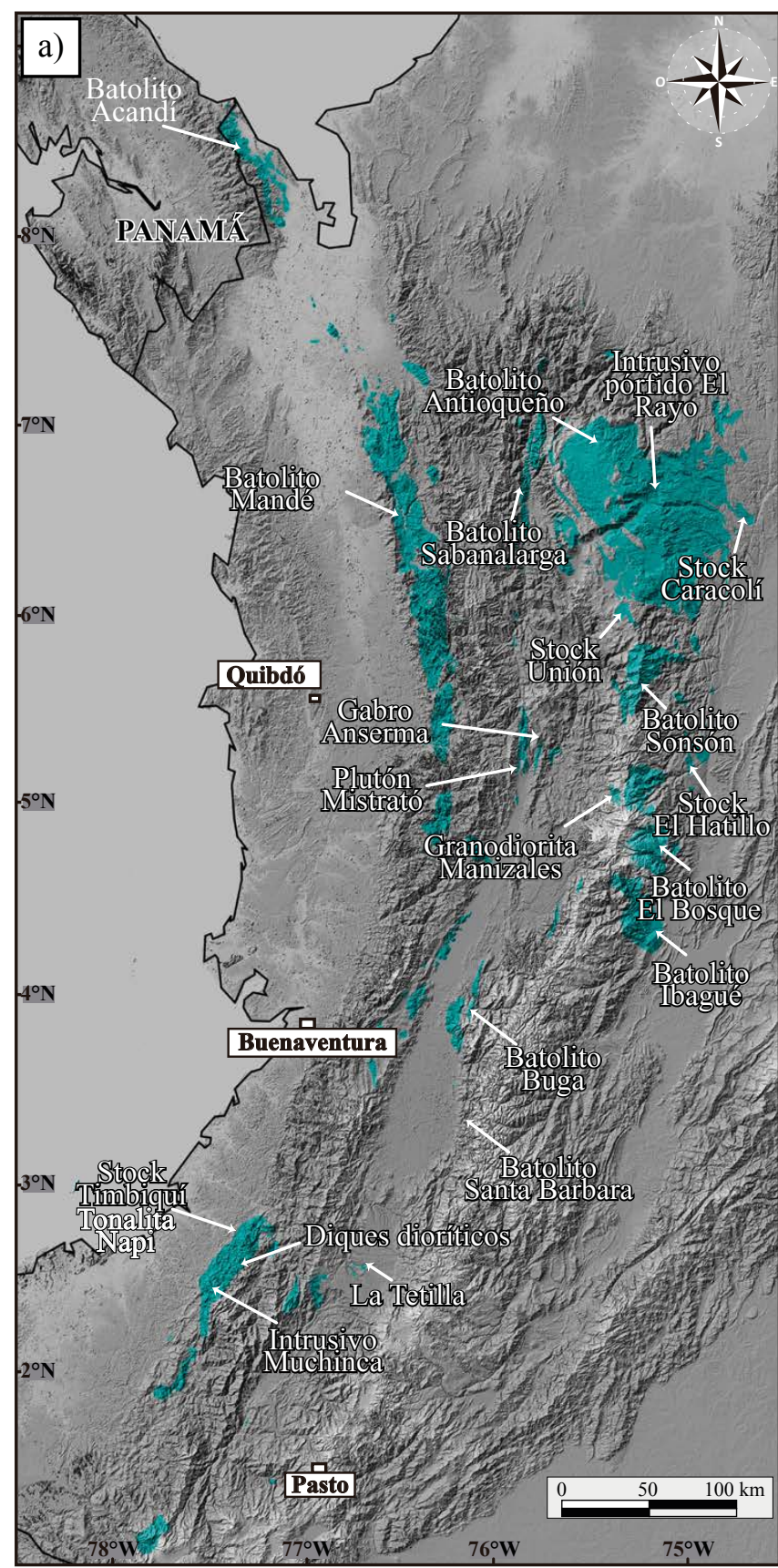

\begin{tabular}{|c|c|c|c|}
\hline \multicolumn{4}{|c|}{ LEYENDA } \\
\hline & $\triangle$ Batolito Acandí & & Batolito Sonsón \\
\hline 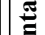 & A Batolito Mandé & $\bar{T}$ & Batolito Antioqueño \\
\hline שِ & A Plutón Mistrató & 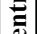 & Granodiorite Manizales \\
\hline 苋 & $\triangle$ La Tetilla & 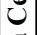 & Stock Unión \\
\hline ฐ & $\triangle$ Stock Córdoba & 吾 & Stock Hatillo \\
\hline : & $\begin{array}{l}\text { Intrusivo Muchinca } \\
\text { y diques dioríticos }\end{array}$ & 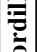 & Batolito Bosque \\
\hline Uे & $\triangle$ Tonalita Napi y & & $\square$ Batolito de Buga \\
\hline _ & Stock Timbiquí & & \\
\hline
\end{tabular}

Figura 4. a) Localización general de las unidades litológicas del evento magmático Paleoceno - Eoceno. b) Diagrama de concentración de álcalis total vs. SiO ${ }_{2}$ (TAS). c) Diagrama de clasificación de $\mathrm{SiO}_{2}$ vs. $\mathrm{K}_{2} \mathrm{O}$. d) Diagrama AFM (A: álcalis $\left.\left(\mathrm{Na}_{2} \mathrm{O}+\mathrm{K}_{2} \mathrm{O}\right), \mathrm{F}: \mathrm{FeO}+\mathrm{Fe}_{2} \mathrm{O}_{3}, \mathrm{M}: \mathrm{MgO}\right)$. Los valores se presentan en \% en peso. Para las rocas distribuidas en la Cordillera Occidental, los análisis son representados por triángulos de distintos colores según la unidad geológica. Los cuadrados representan los análisis realizados en intrusivos localizados a lo largo de la Cordillera Central. Son incluidos los datos reportados en la literatura (Sillitoe et al., 1982; Restrepo et al., 1991; INGEOMINAS, 1979, 1999, 2002a, 2010b; López et al., 2006; Vallejo et al., 2011; Villagómez y Spikings, 2013; Bustamante et al., 2016). 
a)
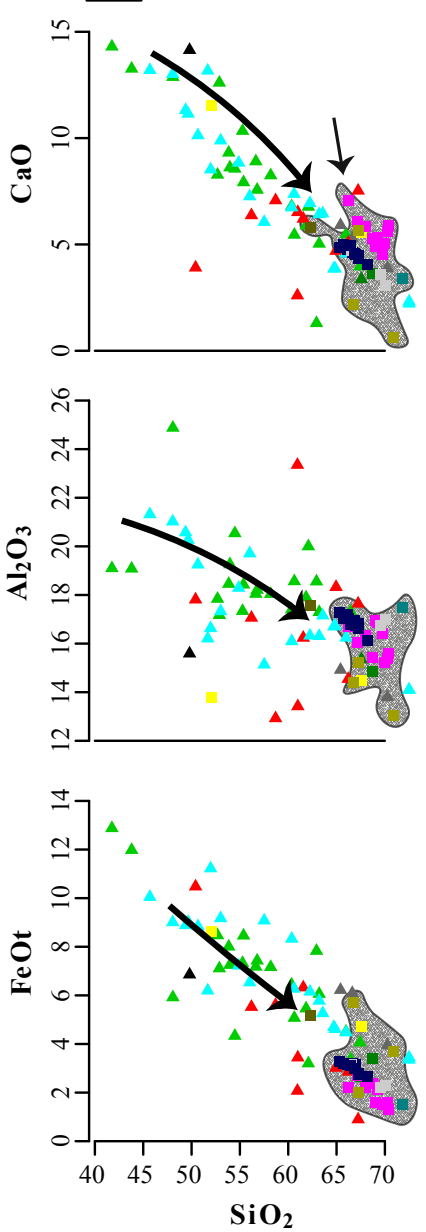
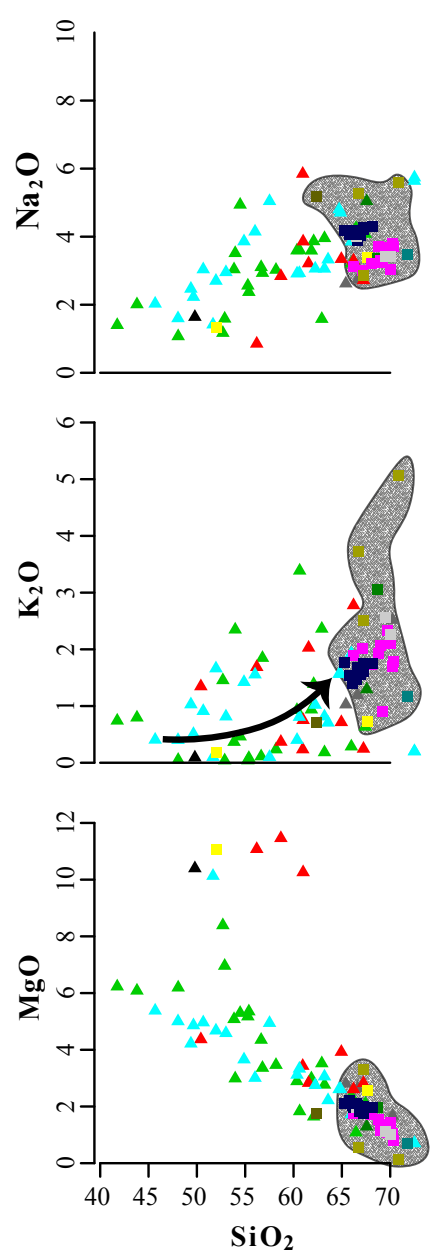

b)
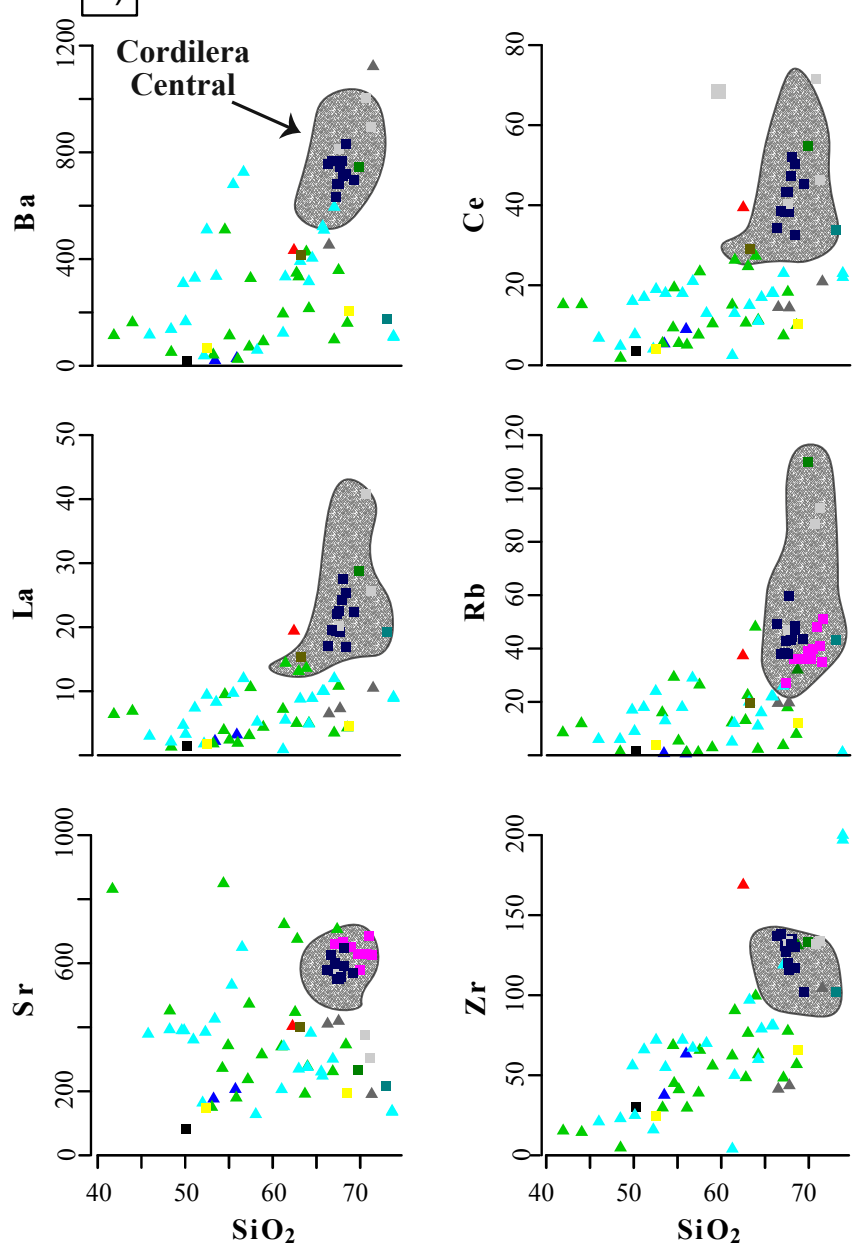

\begin{tabular}{|c|c|c|c|c|c|}
\hline \multicolumn{6}{|c|}{ LEYENDA } \\
\hline ن & $\begin{array}{l}\Delta \text { Batolito Acandí } \\
\Delta \text { Batolito Mandé } \\
\Delta \text { Plutón Mistrató } \\
\Delta \text { La Tetilla }\end{array}$ & $\begin{array}{l}\triangle \text { Stock Córdoba } \\
\text { Intrusivo Muchinca } \\
\text { y diques Dioríticos } \\
\text { Tonalita Napi y } \\
\text { Stock Timbiqui }\end{array}$ & 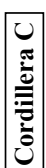 & $\begin{array}{l}\text { Batolito Sonsón } \\
\text { Batolito Antioqueño } \\
\text { Granodiorite Manizales } \\
\text { Stock Unión }\end{array}$ & $\begin{array}{l}\square \text { Stock Hatillo } \\
\text { Batolito Bosque } \\
\text { Gabro Anserma } \\
\square \text { Batolito de Buga }\end{array}$ \\
\hline
\end{tabular}

Figura 5. a) Diagramas de variación en \%wt de elementos mayoritarios $v s \mathrm{SiO}_{2}$. Obsérvese la variación en la concentración de los diferentes óxidos mayoritarios para los cuerpos intrusivos distribuidos en la CC y CO. b) Diagramas de variación de elementos traza (ppm) vs. SiO 2 (\%wt). Para los análisis realizados sobre intrusivos distribuidos sobre la Cordillera Occidental se dibujan triángulos, para los de la Cordillera Central se usan cuadrados y aparecen encerrados en zonas sombreadas.

desarrollo de fracturas extensionales con dirección $\mathrm{NE}$, con rotaciones progresivas en sentido horario (Figura 8c). Bajo estas condiciones se desarrollarían zonas de menor presión, o de fracturas tensionales, que pudieron controlar el emplazamiento elongado de los cuerpos plutónicos sobre la $\mathrm{CO}$.

A lo largo de la CO los intrusivos son alargados, con sus ejes mayores en dirección NE, paralelos al esfuerzo principal $\sigma 1$ inferido y al vector de desplazamiento de la placa Farallón, como se muestra en la Figura 8a. Por esta razón, se puede inferir que la margen continental estuvo sometida a transtensión derecha como mecanismo de primer orden y se minimizan los efectos compresionales. Una explicación para la orientación anómala de algunos batolitos que no se ajustan al esquema descrito, como es el caso del batolito de Mandé, es que fueron rotados (rotación antihoraria) durante los pulsos de la fase Andina.

En contraste, para el mismo periodo Paleoceno - Eoceno, los intru- sivos emplazados en la CC son batolitos y stock con geometrías que tienden a ser circulares en su proyección de planta. Esas geometrías están relacionadas a sistemas extensionales radiales, permitiendo un proceso de emplazamiento magmático con mayor tiempo de permanencia. El emplazamiento de cuerpos intrusivos durante el Paleoceno - Eoceno en ambas cordilleras estuvo controlado por diferencia en magnitud y orientación de las zonas de extensión. Este periodo de tiempo (60.7-53 Ma) está relacionado con altos volúmenes de emplazamiento magmático y a su vez, con los menores valores de velocidad de convergencia entre las placas Farallón y Sudamericana; en consecuencia, las bajas velocidades y la alta oblicuidad de la convergencia configuran la relación entre el desarrollo de fracturas y zonas de debilidad, y facilita el emplazamiento de los magmas.

Los regímenes tectónicos transtensional-extensional pueden ser desarrollados en diferentes panoramas de interacción entre las placas 


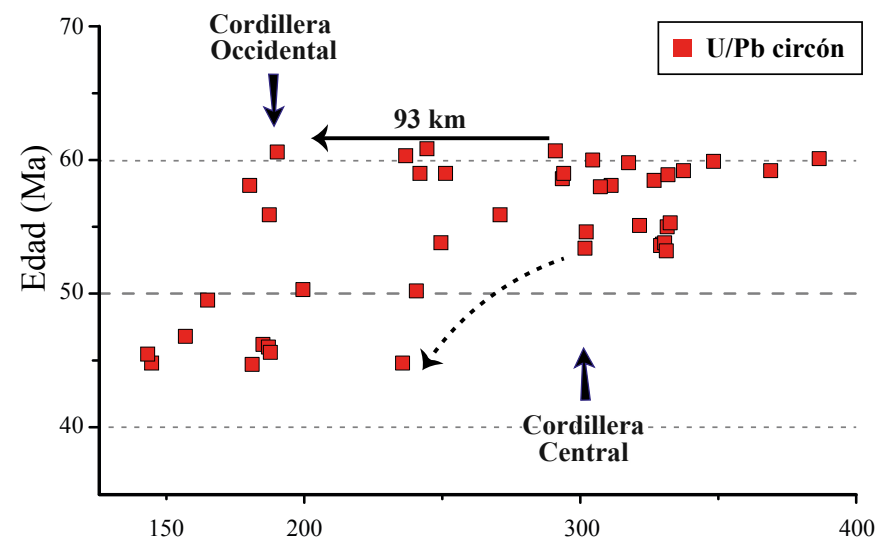

Distancia en kilómetros desde la trinchera

Figura 6. Distribución de las edades de cristalización a partir del método $\mathrm{U} / \mathrm{Pb}$ en circón del occidente colombiano, en las rocas ígneas del Paleoceno - Eoceno en función de la distancia a la fosa actual. La distancia es medida desde la fosa hacia los puntos de muestreo de manera perpendicular, teniendo en cuenta la geometría de las estructuras mayores (Cordillera Central y Occidental).

tectónicas. En sistemas de subducción, es controlado principalmente por la migración negativa (alejamiento) de la trinchera con respecto a la placa subducente (rollback negativo) y por condiciones oblicuas de convergencia. Este proceso hace que la velocidad de subducción sea más rápida que el índice de convergencia, lo que implica una disminución en el acople entre las placas y en la presión de confinamiento, promoviendo el desarrollo de sistemas extensional-transtensional de primer orden en la placa subducente (Daly, 1989; Doglioni et al., 1999, Doglioni et al., 2006a; Doglioni et al., 2007; Schellart, 2008a, 2008b). Otra consecuencia del rollback negativo es el aumento en el ángulo de subducción (Doglioni et al., 2006b; Schellart, 2008b), lo que induciría a una migración de la cuña astenosférica consecuentemente con su arco magmático (subplacado), hacia el borde de la placa subducente, sin embargo, el magmatismo tendría una firma principalmente de manto hidratado (Ramos, 2009).

El régimen transtensivo-extensional es también generado en un borde continental (zona de transición). El magmatismo emplazado allí es favorecido por fallas normales, que posiblemente actúan como zonas permeables para el ascenso de basaltos syn-extensionales, con afinidades oceánicas (MORB-type), que puede estar acompañado por sucesiones de sedimentos oceánicos (Decarlis et al., 2018). En la cercanía límite océano-continente la generación de fallas normales profundas favorece el emplazamiento del subplacado, compuesto de rocas mantélicas principalmente, con posteriores procesos de serpentinización (Whitmarsh et al., 1996; Boillot y Froitzheim, 2001; Desmurs et al., 2001; Decarlis et al., 2018; Ribes et al., 2019; Chloé et al., 2017). Adicionalmente, se desarrollan estructuras extensionales simétricas y asimétricas, que localmente pueden presentar componentes de transcurrencia de segundo y tercer orden de jerarquía, y que controlan la sedimentación (Zhang et al., 2019; Osmundsen y Ebbing, 2008; Chloé et al., 2017). En el caso del borde continental colombiano durante el Paleoceno-Eoceno, o incluso desde el Cretácico (Jaramillo et al., 2017), la interacción de las placas Farallón y Sudamericana fue principalmente transcurrente, como se muestra en la Figura 7.

Un sistema de subducción oblicua con acreción de terrenos como el descrito en el marco tectónico regional habría dejado evidencias características de dicho proceso, a saber: 1 . Un evento magmático Paleoceno-Eoceno en la placa subducente, con la geometría de sus ejes mayores dispuestos en una dirección perpendicular a la dirección de convergencia, que sería continuo hasta la acreción miocénica de terrenos. 2. Los límites de los terrenos acrecionados durante el Mioceno no han sido definidos y no se ha documentado la deformación generada a partir de esa acreción; así mismo, no se evidencia un prisma acrecional al oriente del bloque Panamá-Chocó. 3. No se tiene identificada la fosa del sistema de subducción que trasportó el bloque Panamá-Chocó.

Considerando los problemas expuestos en el párrafo anterior, de un modelo de acreción de terrenos, en este trabajo favorecemos un modelo alternativo retomando el postulado original de Barrero (1979). En este modelo se considera un borde de margen continental fracturado en extensión-transtensión, que facilitó y controló el emplazamiento del evento magmático desde el Cretácico Tardío como lo describió Barrero (1979), hasta el Paleoceno - Eoceno como es reportado aquí (Figura 9).

Un esquema de la evolución y emplazamiento de los magmas en el occidente colombiano, es presentado en la Figura 10, donde se muestra el desarrollado en un margen continental fracturado, previo a la evolución del sistema de subducción. Durante el lapso $60.7-53 \mathrm{Ma}$ se considera una dirección de convergencia de $25^{\circ}$ de azimut y una velocidad de $5 \mathrm{~cm} /$ año, y para el lapso $53 \mathrm{Ma}$ y 44 Ma una dirección de convergencia de $40^{\circ}$ de azimut y una velocidad de $15-20 \mathrm{~cm} /$ año.

\section{Implicaciones geoquímicas en la migración y el emplazamiento de intrusivos}

Los parámetros físico-químicos que dominan el ascenso de los magmas son su densidad y su viscosidad. Así mismo, el mecanismo principal por el cual se genera el ascenso de magma es la propagación de fracturas, las cuales permiten una transferencia rápida de magma y el establecimiento de una red de estructuras que vuelve permeable a la corteza y que facilita el ascenso (Brown, 2004). El incremento de la densidad en los magmas es dado por la adición de $\mathrm{FeO}, \mathrm{MgO} \mathrm{TiO}_{2} \mathrm{y}$ $\mathrm{CaO}$ al fundido, mientras que la adición de álcalis y volátiles produce el efecto contrario (Spera, 2000; Stolper y Walker, 1980). El magmatismo que migró hacia el occidente colombiano durante el Paleoceno-Eoceno es de magmas principalmente básicos e intermedios con afinidades toleíticas, que varían progresivamente a calcoalcalinos. Comparados con los cuerpos distribuidos en la CC contienen mayores concentraciones de $\mathrm{CaO}, \mathrm{FeO}, \mathrm{MgO}$ y una menor concentración de los elementos traza (Rb, Sr, La, Zr, Ce y Ba) (Figura 4 y 5). Por lo anterior, podemos inferir que los magmas de la CO tuvieron densidad relativamente alta $\mathrm{y}$ viscosidad baja, por eso requirieron de fracturas tensionales que facilitaron su ascenso rápido en el borde continental. Progresivamente esos magmas fueron adquiriendo afinidades calcoalcalinas (Figura 8 y 9). El espesor de la corteza en la que se emplazan los magmas juega un papel muy importante. Chiaradia (2015), Chapman (2015) y Profeta et al. (2015) establecen campos en el gráfico Sr/Y vs. MgO que corresponden a espesores corticales promedio (Figura 11). El espesor de la corteza donde se emplazó el evento magmático Paleoceno - Eoceno es inferido por las variaciones en la razón $\mathrm{Sr} / \mathrm{Y}$ y la concentración de $\mathrm{MgO}$ de 47 muestras tomadas en intrusivos ubicadas en la CO. Las muestras presentan valores de $\mathrm{Sr} / \mathrm{Y}$ que varían a lo largo de todo el eje vertical del gráfico, aunque la mayor concentración se ubica en el rango donde Chiaradia (2015) establece un espesor de corteza entre $<20-30 \mathrm{~km}$ (Figura 11). Las muestras que presentan mayores valores de $\mathrm{Sr} / \mathrm{Y}$, estarían relacionadas a las fases finales de emplazamiento. En la CC, por el contrario, los análisis marcan claramente un aumento del valor $\mathrm{Sr} / \mathrm{Y}$ con la disminución del MgO, lo que permite interpretar espesores de la corteza continental mayores a $30 \mathrm{~km}$ (Figura 11). Esos espesores mayores son consistentes con las composiciones ácidas de series calco-alcalinas provenientes de magmas más evolucionados y con mayor tiempo de permanencia.

Así entonces, el emplazamiento de los magmas de la CO está condicionado por su mayor densidad y baja viscosidad, en un medio 

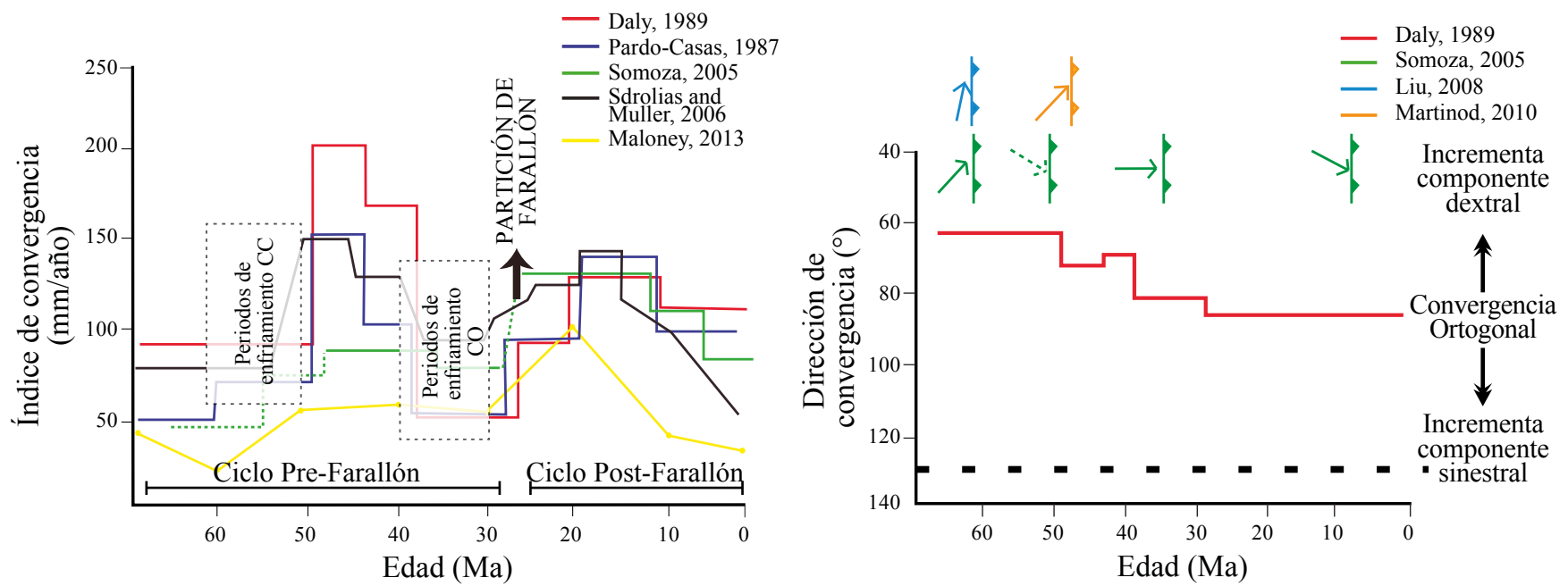

Figura 7. a) Velocidad y ángulo de convergencia de la placa Farallón - Nazca - Coiba y la placa Sudamericana para el Cenozoico. Distribución de los máximos periodos de enfriamiento según Noriega-Londoño et al. (2019), para los cuerpos plutónicos/volcánicos ubicados en la Cordillera Central y la Cordillera Occidental.

de propagación de fracturas controladas por un régimen tectónico transtensional, desarrollado en una corteza de borde continental con un espesor $<25-30 \mathrm{~km}$.

\section{EVOLUCIÓN HACIA UN EQUILIBRIO DINÁMICO DEL MARGEN PACÍFICO COLOMBIANO}

Como se ilustra en la Figura 7, entre 40 Ma y 26 Ma tuvo lugar una disminución significativa en la velocidad de convergencia de la placa Farallón, de $20 \mathrm{~cm} /$ año a $5 \mathrm{~cm} /$ año, con un ángulo promedio de convergencia de $30^{\circ}$ de azimut, (Pardo-Casas y Molnar, 1987; Daly, 1989; Maloney et al., 2013; Sdrolias and Müller, 2006; Somoza y Chidella, 2005; Martinod et al., 2010; Liu et al., 2008). El cambio en la velocidad de la placa Farallón y la disminución de la velocidad total de la placa Sudamericana reduce la velocidad de convergencia y se espera una disminución en la eficiencia del acople entre las placas. Al reducir el acople, se disminuye la carga tectónica y, en consecuencia, el orógeno reduce su tasa de levantamiento. El proceso de equilibrio de masas da lugar a un aumento relativo en la remoción por procesos gravitacionales de la masa orogénica y de la sedimentación en los sistemas cuencales intramontanos. Este proceso de relajación del esfuerzo tectónico regional se denomina en este estudio Equilibrio Dinámico, de acuerdo al principio físico de d'Alembert.

En el ambiente intra-continental Andino colombiano, el equilibrio dinámico es manifestado por la discordancia progresiva del Eoceno medio/tardío a lo largo de las cuencas sedimentarias del Valle del Cauca Patía, Valle del Magdalena, Sinú San Jacinto entre otras, sumada a procesos masivos de peneplanización para los altos topográficos ubicados en el norte de Colombia relacionados a los procesos de la discordancia (Barrero, 1979; Cediel et al., 1998). Es también reportado en las cuencas de Yucatán (México), en Venezuela y el Caribe colombiano (James, 2009). Durante ese periodo, se reactivaron los piedemontes cordilleranos, promoviendo el depósito de secuencias de abanicos de las Formaciones Chimborazo y Peña Morada, seguida por la Formación Guachinte-Ferreira en la Cuenca Cauca Patía (Barrero et al., 2007; Ecopetrol, 2000), Formaciones San Cayetano y Maco en las cuencas Valle Inferior del Magdalena (VIM) (Pozo Betulia-1 y el Pozo ANH-Plato-1X) y Sinú San Jacinto (SSJ) (Barrero et al., 2007; $\mathrm{ANH}, 2017)$, y para la cuenca Valle Medio del Magdalena (VMM) en las formaciones Lizama, Esmeralda, La Paz (Gómez et al., 2005).

En los ambientes de plataforma continental, el equilibrio dinámico es evidenciado por la distribución de secuencias de calizas arrecifales y su variación de facies laterales, en toda la margen Atlántica y Pacífica colombiana. Los ambientes de plataformas de carbonatos predominaron durante el Eoceno medio-tardío, a lo largo de las márgenes Pacífica y Caribe de Colombia (pozos: ANH-P27; ANH-P17; ANH-Nueva Esperanza-1X; ANH-San Antero-1; ANH-La Estrella-1X) (ANH-GRP, 2014; ANH, 2017; Grajales et al., 2016), Venezuela y Yucatán (James, 2009). Estos sistemas están reportados igualmente en la Península Azuero y en el área Coiba al sur de Panamá, donde las secuencias abarcan edades desde el Eoceno temprano al Eoceno medio (Buchs et al., 2011). También se reportan al sur de Costa Rica, por las Formaciones Caliza Silícea de Buenavista, Calizas Punta Quepos y Caliza Punta Cuevas (Baumgartner et al., 1984). Adicionalmente, se han reportado al norte de Ecuador, en la Cuenca Esmeraldas por secuencias clásticas carbonáticas de la Formación Azúcar.

Durante el Oligoceno temprano es reportada una discordancia transgresiva en las cuencas intramontanas y de borde continental (Barrero et al., 2007). A partir de los 44 Ma y hasta los $26 \mathrm{Ma}$, no se han reportado edades de cristalización de cuerpos intrusivos/volcánicos en el occidente colombiano, determinándose así un gap magmático de 18 millones de años. Estos dos procesos podrían estar relacionados a las fases finales del equilibrio dinámico o fases iniciales de la reactivación orogénica.

La edad reportada para el siguiente evento magmático Oligoceno - Mioceno temprano es de 23 Ma y fue establecida en el Stock de Piedrancha en el sector sur de la CO, a partir de los métodos K/Ar en biotita (Álvarez et al., 1981).

\section{CONCLUSIONES}

El emplazamiento de plutones del evento magmático Paleoceno - Eoceno en la margen Pacífica colombiana es favorecido por un régimen tectónico transtensional, el cual se desarrolló sobre una margen continental fracturada, previa a la evolución del sistema de subducción. La geometría elongada de los cuerpos plutónicos presenta su eje mayor paralelo a la dirección del esfuerzo principal $\sigma 1$, por lo que se infiere que las fracturas y zonas de debilidad permitieron el ascenso 

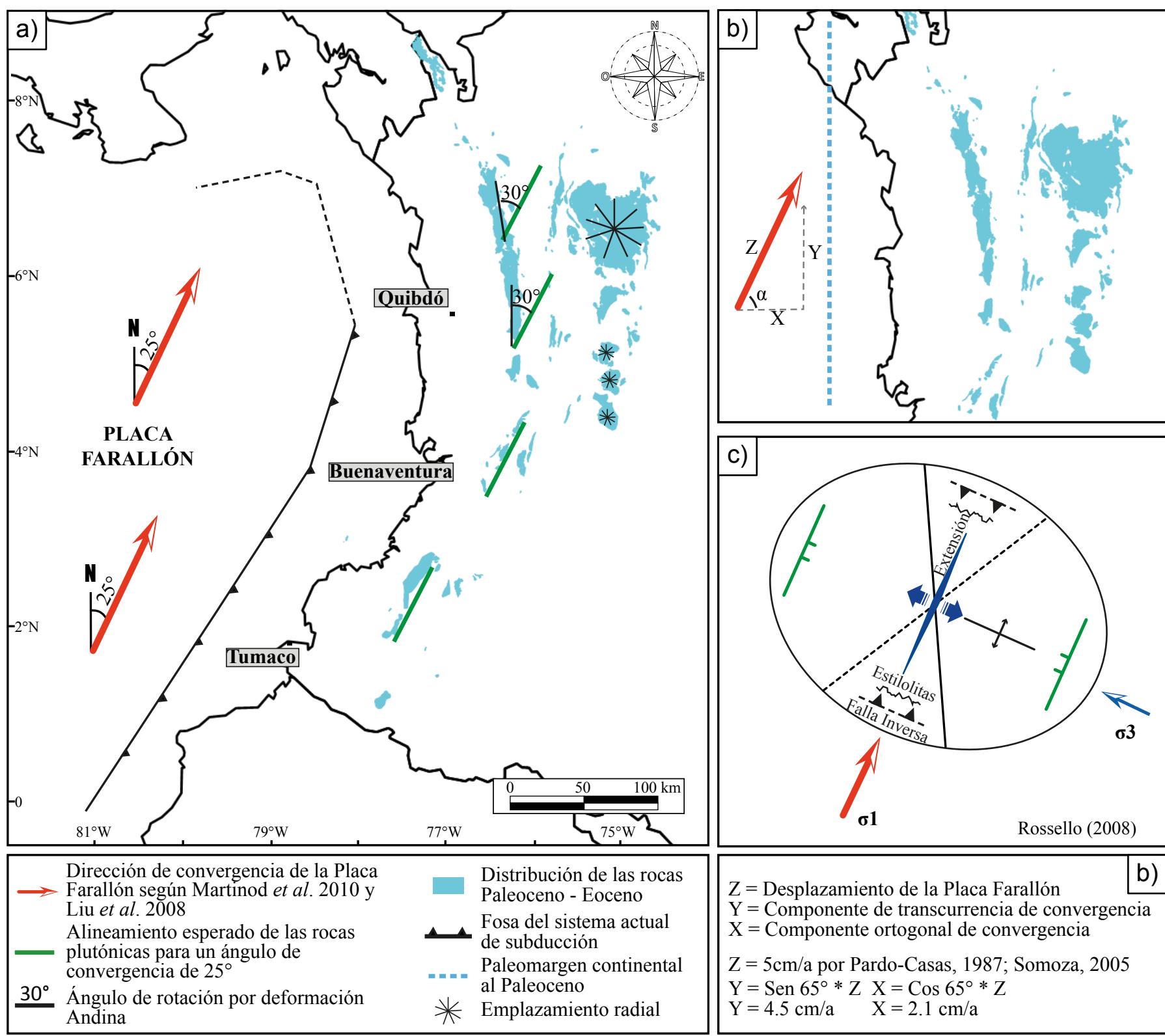

c)

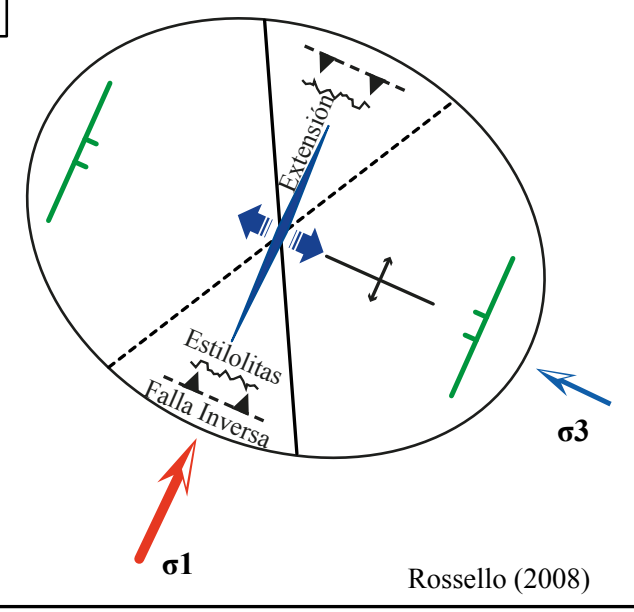

$\mathrm{Z}=$ Desplazamiento de la Placa Farallón

$\mathrm{Y}=$ Componente de transcurrencia de convergencia $\mathrm{X}=$ Componente ortogonal de convergencia

$\mathrm{Z}=5 \mathrm{~cm} / \mathrm{a}$ por Pardo-Casas, 1987; Somoza, 2005

$\mathrm{Y}=\operatorname{Sen} 65^{\circ} * \mathrm{Z} \mathrm{X}=\operatorname{Cos} 65^{\circ} * \mathrm{Z}$

$\mathrm{Y}=4.5 \mathrm{~cm} / \mathrm{a} \quad \mathrm{X}=2.1 \mathrm{~cm} / \mathrm{a}$

Figura 8. a) Disposición geométrica de las rocas plutónicas con su eje mayor en una dirección NE representada por las líneas continuas de color verde. En el área norte, si se hace la corrección por la rotación horaria $\left(30^{\circ}\right)$ de los cuerpos intrusivos, en su azimut regional, el eje mayor de todos los cuerpos intrusivos de este evento magmático, son paralelos a las flechas rojas. La dirección de desplazamiento de la placa Farallón es simbolizada por la flecha roja, la cual es paralela a la dirección del esfuerzo compresivo máximo $\sigma 1$. b) Partición del vector de desplazamiento de la placa Farallón. La línea punteada denominada X, representa la componente ortogonal del desplazamiento y la línea punteada denominada Y, la componente transcurrente del desplazamiento. La línea azul punteada hace referencia al límite de la margen continental en el Paleoceno. c) Elipse de deformación generada para $\sigma 1$ paralelo al vector de desplazamiento en dirección NE ( $25^{\circ}$ de azimut) durante el Paleoceno - Eoceno. Las flechas azules indican la dirección del esfuerzo principal mínimo $\sigma 3$, la línea azul continua, la dirección esperada del desarrollo de fracturas extensionales y tensionales en segundo orden de deformación.

de cuerpos intrusivos. Las edades de cristalización $\mathrm{U} / \mathrm{Pb}$ muestran que la provincia magmática se emplazó de $60 \mathrm{Ma}$ a $53 \mathrm{Ma}$ al norte de las cordilleras Central y Occidental. En la Cordillera Occidental se encuentra el Batolito de Mandé y en la Cordillera Central pulsos magmáticos del Batolito Antioqueño. A partir de los 53 Ma tuvo lugar una migración del arco magmático hacia el occidente, hasta los $44 \mathrm{Ma}$ los cuerpos intrusivos más jóvenes son principalmente básicos e intermedios con afinidades toleíticas y se emplazaron rápidamente en un borde continental (corteza transicional) con un espesor $<25-30 \mathrm{~km}$. La composición de los magmas cambió progresivamente a ser cal- coalcalinas. La continuidad del cinturón de intrusivos en la Cordillera Occidental es documentada hasta el sur en el límite Colombia-Ecuador.

La disminución significativa en la velocidad de convergencia entre los 40 \pm 5 Ma y 26 Ma dio lugar a un proceso denominado Equilibrio Dinámico sobre la margen pacífica colombiana. El equilibrio dinámico es reflejado en distintos ambientes sedimentarios continentales: en ambientes de plataforma continental, por una extensa sedimentación de secuencias clásticas carbonáticas de edad Eoceno Medio-Tardío a lo largo de la margen Pacífica y Caribe de Colombia, Ecuador, Panamá y Costa Rica; en ambientes intra-continentales por la discordancia 


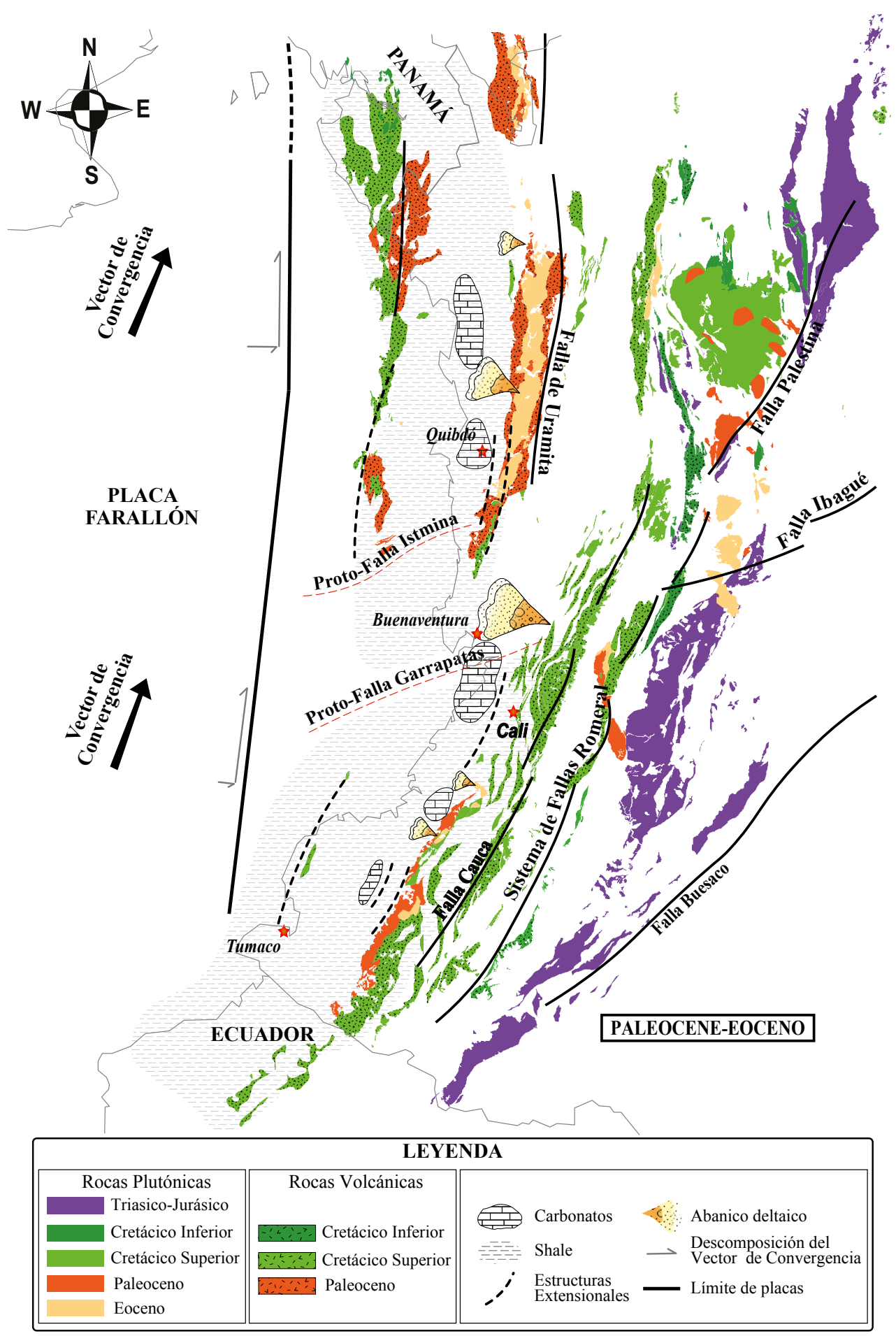

Figura 9. Representación paleogeográfica del emplazamiento de los cuerpos plutónicos y volcánicos durante el Paleoceno-Eoceno. Las rocas ubicadas al norte de la Proto-Falla de Istmina y al occidente de la falla de Uramita aparecen en su posición en el Paleoceno-Eoceno. Se reconstruyó su posición a partir de la corrección por la rotación generada en la deformación andina. Obsérvese cómo los cuerpos ígneos del Cretácico Superior, principalmente volcánicos (basaltos y dacitas), y las fallas principales Romeral, Cauca y Uramita, también presentan su eje principal paralelo al vector de convergencia. Las líneas punteadas en negro representan estructuras extensionales, interpretadas en líneas símicas y secciones estructurales (Grajales et al., 2016). El Triásico-Jurásico está conformado por plutones de sienogranitos que varían de tonalitas y cuarzomonzonitas a cuarzomonzodioritas. El Cretácico Inferior presenta plutones de gabro y peridotita serpentinizados, los volcánicos son basaltos y andesitas intercalados con lodolitas carbonosas y arenitas (Complejo Quebradagrande). El Cretácico Superior es conformado por granodioritas, tonalitas y cuarzodioritas, localmente gabros, que varía a cuarzogabros; las rocas volcánicas son compuestas por basaltos toleíticos, doleritas, picritas intercaladas con tobas básicas. El Paleoceno-Eoceno son tonalitas, granodioritas, cuarzodioritas y localmente gabros, las rocas volcánicas son basaltos intercalados con chert, lodolitas calcáreas y arenitas calcáreas, ocasionalmente calizas. Durante el Paleoceno-Eoceno la sedimentación en la plataforma está dada por secuencias continuas de shale marino con desarrollo de carbonatos. Localmente, hay abanicos deltaicos progradando hacia la plataforma en extensión. 


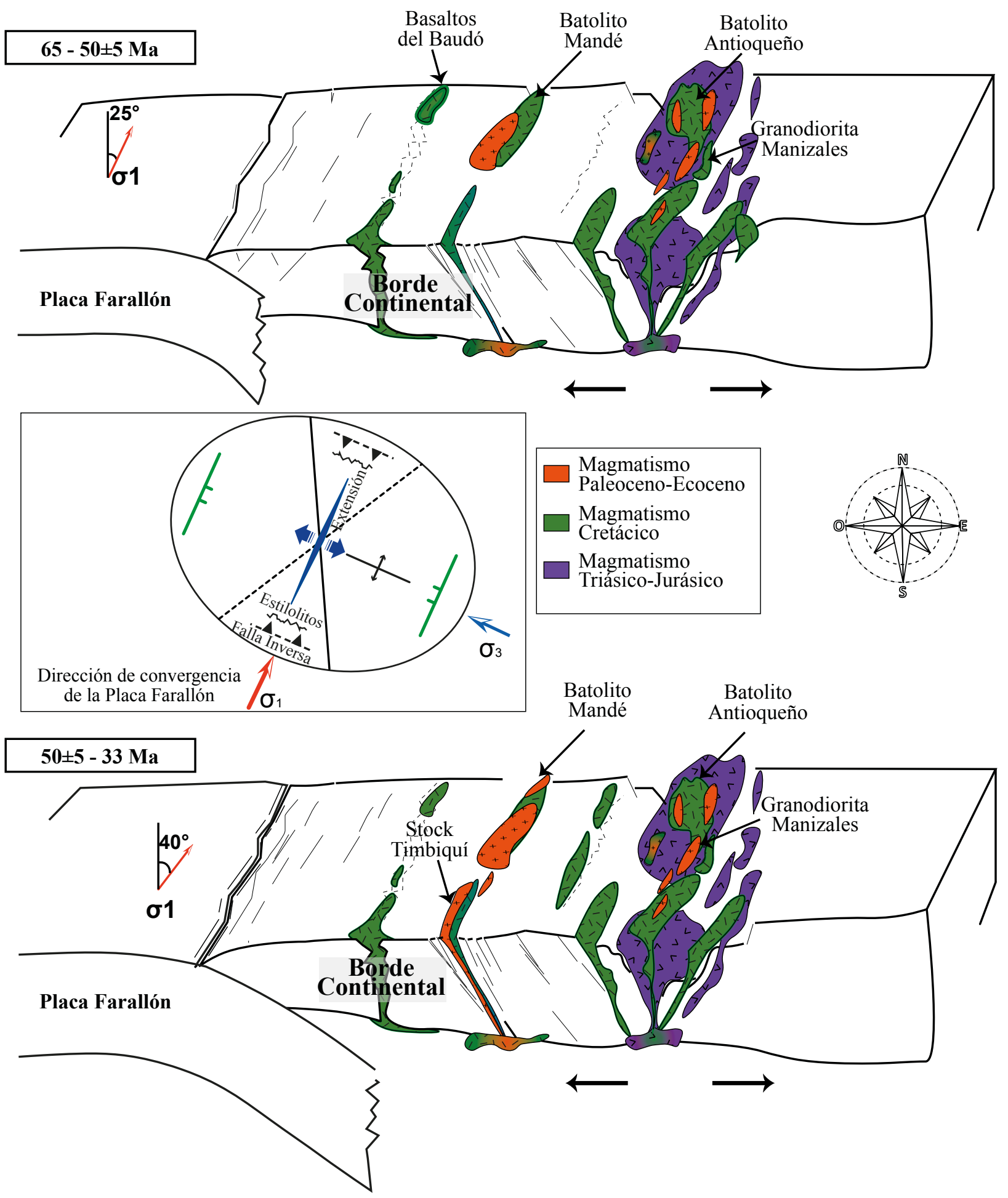

Figura 10. Reconstrucción esquemática de la evolución y emplazamiento de rocas plutónicas del evento magmático durante el Paleoceno-Eoceno en el occidente colombiano. El gráfico superior representa el emplazamiento durante los $65-50$ Ma con una dirección de convergencia de $25^{\circ}$ de azimut y una velocidad de $5 \mathrm{~cm} / \mathrm{a}$. Se muestra la elipse de deformación correspondiente al tensor de esfuerzos mayor, que representa la dirección de convergencia (flecha roja). Las líneas verdes dentro de la elipse simbolizan la tendencia de las fracturas tensionales dentro del sistema. El gráfico inferior representa el emplazamiento durante 50 a $33 \mathrm{Ma}$. con una dirección de convergencia de $40^{\circ}$ de azimut y una velocidad de $15-20 \mathrm{~cm} / \mathrm{a}$. Los cuerpos del evento magmático (azules), migran hacia el occidente durante este periodo. 

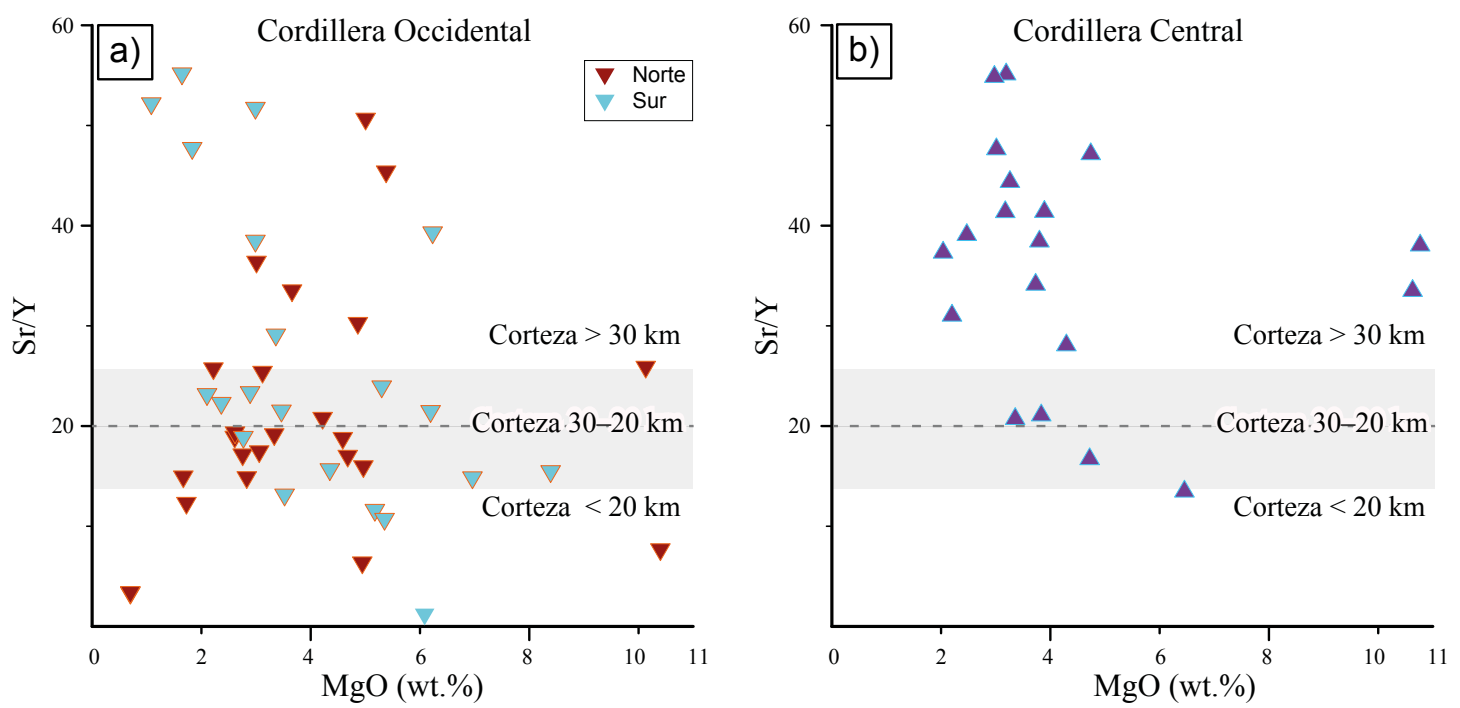

Figura 11. Gráficas Sr/Y vs. MgO de 89 análisis recopilados de la CC y CO, que muestra espesores promedio de la corteza según Chiaradia (2015), Chapman (2015) y Profeta et al. (2015), en la que se emplazaron estos cuerpos. a) Análisis realizados en cuerpos intrusivos de la CO (57 muestras). Los triángulos en celeste representan los cuerpos ubicados en el sector sur de la cordillera, como son el Stock de Timbiquí, la Tonalita de Napí, Diques Dioríticos e intrusivo de Munchica. Los triángulos rojos, los intrusivos ubicados al norte de la cordillera, Batolito de Mande y Batolito Acandí. b) Análisis de cuerpos intrusivos ubicados a $5^{\circ} \mathrm{N}$ sobre la CC (32 muestras).

progresiva regional del Eoceno medio, y la peneplanización de altos topográficos al norte de Colombia, que favorecieron la exhumación de las cúpulas de los plutones, y refleja la reactivación de los piedemontes cordilleranos con progradaciones de abanicos.

\section{AGRADECIMIENTOS}

El presente trabajo de investigación fue realizado con el apoyo de la Agencia Nacional de Hidrocarburos (ANH), que dispuso de una amplia base de datos para su desarrollo. Agradecemos a dos árbitros anónimos por sus valiosas recomendaciones y sugerencias.

\section{MATERIAL SUPLEMENTARIO}

Las Tablas S1, S2 y S3 se pueden descargar en el portarl web de la Revista $<$ http://rmcg.unam.mx/>, en la versión html del resumen de de este artículo.

\section{REFERENCIAS}

ANH (Agencia Nacional de Hidrocarburos), 2017, Broshure Técnico. Procedimiento competitivo Sinú - San Jacinto: Bogotá, Colombia, Agencia Nacional de Hidrocarburos, Informe Especial, 109 pp. <https:// www.anh.gov.co/Asignacion-de-areas/San-Jacinto/Documentos\%20 compartidos/Terminos\%20Referencia\%20Procedimiento\%20Sinu $\% 20$ San\%20Jacinto\%202017.pdf>, consultado: noviembre de 2018.

ANH-GRP (Agencia Nacional de Hidrocarburos, Geología Regional y Prospección), 2014, Cartografía geológica a escala 1:100.000 de las planchas 340, 362, 385 y 409 localizadas en la Cuenca de Tumaco, así como el inherente levantamiento de columnas estratigráficas y muestreo litológico para los análisis petrográficos, bioestratigráficos, geoquímicos, petrofísicos y radiométricos: Bogotá, Colombia, Agencia Nacional de Hidrocarburos, Publicación Especial, 207 pp. <http://recordcenter.sgc. gov.co/B14/23008010024581/documento/pdf/2105245811101000.pdf> consultado: noviembre de 2018.
ANH-UCaldas (Agencia Nacional de Hidrocarburos, Universidad de Caldas), 2011, Estudio integrado de los núcleos y registros obtenidos de los pozos someros (slim holes) perforados por la ANH: Manizales, Colombia, Agencia Nacional de Hidrocarburos, Publicación Especial, 73 pp. $<$ (PDF) Estudio integrado de los núcleos y registros obtenidos de los pozos someros tipo "slim holes" en la Cuenca Sinú-TOMO 1 | Hermann Bermúdez Academia.edu>, consultado: septiembre de 2018.

Álvarez, J., Orrego, A., Botero, G., Linares, E., 1981, Determinación de edad K/ Ar del Stock de Suárez, Cauca: Boletín de Ciencias de la Tierra, 5/6, 39-43.

Aspden, J., McCourt, J., Brook, M., 1987, Geometrical control of subductionrelated magmatism: the Mesozoic and Cenozoic plutonic history of Western Colombia: London, Journal of the Geological Society, 144, 893-905.

Barrero, D., 1979, Geology of the Central Western Cordillera, west of Buga and Roldanillo, Colombia: Publicaciones Geológicas especiales del Ingeominas, 4, 1-75.

Barrero, D., Pardo, A., Vargas, A., Martínez, J., 2007, Colombian Sedimentary Basin: nomenclature, boundaries and petroleum geology, a new proposal: Bogotá, Colombia, Agencia Nacional de Hidrocarburos, Publicación especial, 1-92, <http://www.anh.gov.co/Informacion-Geologica-yGeofisica/Cuencas-sedimentarias/ Documents/colombian_sedimentary_ basins.pdf>, consulta: noviembre 2018 .

Bayona, G., Cardona, A., Jaramillo, C., Mora, A., Montes, C., Valencia, V., Ibañez, M., 2012, Early Paleogene magmatism in the northern Andes: Insights on the effects of Oceanic Plateau-continent convergence: Earth and Planetary Science Letters, 331-332, 97-111.

Boillot, G., Froitzheim, N., 2001, Non-volcanic rifted margins, continental break-up and the onset of sea-floor spreading: some outstanding questions: Geological Society, London, Special Publications, 187, 9-30.

Boschman, L., Hinsbergen, D., Torsvik, T., Spakman, W., Pindell, J., 2014, Kinematic reconstruction of the Caribbean region since the Early Jurassic: Earth-Science Reviews, 138, 108-136.

Brown, M., 2004, The mechanism of melt extraction from lower continental crust of orogens: Transactions of the Royal Society of Edinburgh, 95, 35-48.

Baumgartner, P.O., Mora, C.M., Butterlin, J., Sigal, J., Glacon, G., Azéma, J., Bourgois, J., 1984, Sedimentación y paleogeografía del Cretácico y Cenozoico del litoral Pacífico de Costa Rica: Revista Geológica América Central, 1, 57-136.

Buchs, D., Baumgartner, P., Baumgartner, C., Flores, K., Bandini, A., 2011, Upper Cretaceous to Miocene tectonostratigraphy of the Azuero area (Panamá) and the discontinuous accretion and subduction erosion along the Middle 
American margin: Tectonophysics, 512, 31-46.

Bustamante, C., Cardona, A., Archanjo, C., Bayona, G., Lara, M., Valencia, V., 2016, Geochemistry and isotopic signatures of Paleogene plutonic and detrital rocks of the Northern Andes of Colombia: A record of postcollisional arc magmatism: Lithos, 277, 199-209.

Cardona, A., León, S., Jaramillo, J.S., Montes, C., Valencia, V., Vanegas, J., Bustamante, Echeverri, S., 2018, The Paleogene arcs of the northern Andes of Colombia and Panama: Insights on plate kinematic implications from new and existing geochemical, geochronological and isotopic data: Tectonophysics, 749, 88-103.

Cardona, A., León, S., Jaramillo, J.S., Valencia, V., Zapata, S., Pardo-Trujillo, A., Schmitt, A.K., Mejía, D., Arenas, J.C., 2020, Cretaceous record from a Mariana- to an Andean-type margin in the Central Cordillera of the Colombian Andes, en Gómez, J., Pinilla-Pachon, A.O. (eds.), The Geology of Colombia, Volume 2 Mesozoic: Bogotá, Colombia, Servicio Geológico Colombiano, Publicaciones Geológicas Especiales, 36, 39 pp. https://doi. org/10.32685/pub.esp.36.2019.10.

Cediel, F., Barrero, D., Caceres, C., 1998, Seismic expression of structural styles in the basins of Colombia in six volumes: London, prepared by Geotec for Ecopetrol, Robertson Research, 89 pp.

Cediel, F., Shaw, R., Cáceres, C., 2003, Tectonic assembly of the Northern Andean Block, en C. Bartolini, Buffler, R.T., Blickwede, J. (eds.), The Circum-Gulf of Mexico and the Caribbean: Hidrocarbon habitats, basin formation, and plate tectonics: American Asociation of Petroleoum Geologists (AAPG) Memoir, 79, 815-848.

Chapman, M., 2015, Crustal thickness control on Sr/Y signatures of recent arc magmas: An Earth scale perspective. Scientific Reports: Petrology Geochemistry, 5, 8115.

Chiaradia, M., 2015, Crustal thickness control on Sr/Y signatures of recent arc magmas: an earth scale perspective: Scientific Reports, doi: 10.1038/ srep08115.

Chloé, N., Sylvie, L., Khaled, K., Abdulhakim, A., 2017, Tectono-sedimentary evolution of the eastern Gulf of Aden conjugate passive margins: narrowness an asymmetry in oblique rifting context: Tectonophysics, 721, 322-348, doi: 10.1016/j.tecto.2017.09.024.

Cochrane, R., 2013, U/Pb thermochronology, geochronology and geochemistry of NW South America: Rift to drift transition, active margin dynamics and implications for the volume balance of continents: Ginebra, Suiza, Université de Genève, tesis de doctorado, 191 pp. DOI: 10.13097/ archive-ouverte/unige:30029.

Daly, M., 1989, Correlations between Nazca/Farallon plate kinematics and forearc basin evolution in Ecuador: Tectonics, 8(4), 769-790.

Decarlis, A., Gillard, M., Tribuzio, R., Epin, M.E., Manarschal, G., 2018, Breaking up continents at magma-poor rifted margins: a seismic vs. outcrop perspective: Journal of the Geological Society, 175(6), 875-882, DOI: https://doi.org/10.1144/jgs2018-041.

Desmurs, L., Manatschal, G., Berdoulli, D., 2001, The Steinmann Trinity revisited: mantle exhumation and magmatism along an ocean-continent transition: the Platta nappe, eastern Switzerland: Geological Society, London, Special Publications, 187, 235-266.

Doglioni, C., Harabaglia, P., Merlini, S., Mongeli, F., Paccerillo, A., Piromallo, C., 1999, Orogens and slabs vs. their direction of subduction: Earth Science Reviews, 45, 167-208.

Doglioni, C, Cuffaro, M., Carminati, E., 2006a, What moves Slabs?: Bollettino di Geofisica Teorica ed Applicata, 47 (3), 227-247.

Doglioni, C., Carminati, E., Cuffaro, M., 2006b, Simple Kinematics of Subduction Zones: International Geology Review, 48, 479-496.

Doglioni, C., Carminati, E., Cuffaro, M., Scrocca, D., 2007, Subduction Kinematics and dynamic constraints: Earth-Science Reviews, 83, 125-175.

Duque-Caro, H., 1990, The Choco Block in the northwestern corner of South America: Structural, tectonostratigraphic, and paleogeographic implications: Journal of South American Earth Sciences, 3(1), 71-84.

Echeverri, S., Cardona, A., Pardo, A., Borrero, C., Rosero, S., López, S., 2015, Correlación y geocronología Ar-Ar del basamento Cretácico y el relleno sedimentario Eoceno superior - Mioceno (Aquitaniano inferior) de la cuenca de antearco de Tumaco, SW de Colombia: Revista Mexicana de Ciencias Geológicas, 32(2), 179-189.

Ecopetrol, 2000, Potencial generador del Pacífico colombiano Cuenca San Juan, Biocronología y Paleoambientes: Ecopetrol, Informe Interno, 1-150.

Estrada, J.J., 1995, Paleomagnetism and accretion events in the northern Andes:
Binghamton, State University of New York, USA, tesis de doctorado, 172 pp.

Etayo-Serna, F., Barrero, D., Duue-Caro, H., y otros 1983, Mapa de Terrenos geológicos de Colombia: Bogotá, Colombia, INGEOMINAS, Publicación especial, 14, 1-235.

Gómez, E., Jordan, T.E., Allmendinger, R.W., Hegarty K., Kelley, S., 2005, Syntectonic Cenozoic sedimentation in the northern middle Magdalena Valley Basin of Colombia and implications for exhumation of the Northern Andes: Geological Society of America Bulletin, 117(5-6), 547-569.

Grajales, J.A, Tassara, A., Osorio, J., Ardila , L., Pardo, A., 2016, Nuevas evidencias de un sistema petrolífero activo en la marcen Pacífica Colombiana, en XII Simposio Bolivariano, Exploración Petrolera en las cuencas subandinas: Bogotá, Colombia, Asociación Colombiana de Geólogos y Geofísicos del Petróleo (ACGGP), 5.

Grosser, J.R., 1989, Geotectonic evolution of the Western Cordillera of Colombia: New aspects from geochemical data on volcanic rocks: Journal of South American Earth Sciences, 2(4), 359-369.

INGEOMINAS (Instituto Nacional de Investigaciones Geológico Mineras), 1979, Evaluación del prospecto de cobre y molibdeno en las cabeceras del río Muerto, municipio de Acandí, departamento del Chocó, INGEOMINAS: Colombia, Informe Especial 1799, 99 1-211.

INGEOMINAS (Instituto Nacional de Investigaciones Geológico Mineras), 1999, Catálogo de Unidades Litoestratigráficas de Colombia. Batolito de Mandé, Cordillera Occidental, Departamento de Antioquia, Chocó y Risaralda: Medellín, Colombia, INGEOMINAS.

INGEOMINAS (Instituto Nacional de Investigaciones Geológico Mineras), 2002a, Catálogo de Unidades Litoestratigráficas de Colombia. Granodiorita de Manizalles, Cordillera Central, Colombia: Medellín, Colombia INGEOMINAS.

INGEOMINAS (Instituto Nacional de Investigaciones Geológico Mineras), 2002b, Catálogo de Unidades Litoestratigráficas de Colombia. PaleocenoEoceno, Batolito de Bandé: Medellín, Colombia, INGEOMINAS.

INGEOMINAS (Instituto Nacional de Investigaciones Geológico Mineras), 2002c, Catálogo de las Unidades Litoestratigráficas de Colombia. Cuarzodiorita de Mistrató: Medellín, Colombia, INGEOMINAS.

INGEOMINAS (Instituto Nacional de Investigaciones Geológico Mineras), 2002d, Catálogo de Unidades Litoestratigráficas de Colombia. Gabro de Anserma. Cordillera Occidental: Medellín, Colombia, INGEOMINAS.

INGEOMINAS (Instituto Nacional de Investigaciones Geológico Mineras), 2002e, Catalogo de las Unidades Litoestratigráficas de Colombia. Batolito de Sonsón: Medellín, Colombia, INGEOMINAS.

INGEOMINAS (Instituto Nacional de Investigaciones Geológico Mineras), 2010a, Geologia de las Planchas 58 Capurganá, 68 Acandí y 79 Bis., Cerro Tagarí: Medellin, Colombia, INGEOMINAS.

INGEOMINAS (Instituto Nacional de Investigaciones Geológico Mineras), 2010b , Geoquímica, geocronología de las unidades litológicas asociadas al sistema de Fallas Cauca-Romeral, sector centro-sur. Tomo I. Proyecto Cordillera Occidental: Medellín, Colombia, INGEOMINAS.

Janousek, V., Farrow, C.M., Erban, V., 2006, Interpretation of Whole-rock Geochemical Data in Igneous Geochemistry: Introducing Geochemical Data Toolkit (GCDkit), Journal of Petrology, 47, 1255-1259.

Jaramillo, J., Cardona, A., León, S., Valencia, V., Vinasco, C., 2017, Geochemistry and geochronology from Cretaceous magmatic and sedimentary rocks at $6^{\circ} 35^{\prime} \mathrm{N}$, western flank of the Central Cordillera (Colombian Andes): Magmatic record of arc growth and collision: Journal of South American Eart Sciences, 76, 460-481.

James, H.J., 2009, In situ origin of the Caribbean: Discussion of data, en James, K.H., Lorente, M.A., Pindell, J.L. (eds), The Origin and Evolution of the Caribbean Plate: London, The Geological Society of London Special Publications, 328, 77-125, DOI: 10.1144/SP328.3 0305.

Leal Mejía, H., 2011, Phanerozoic gold metallogeny in the colombian Andes: A tectono/magmatic approach: Barcelona, España, Universitat de Barcelona, tesis doctoral, $1000 \mathrm{pp}$

Lissina, B., 2005, A Profi le through the Central American Landbridge in Western Panama: 115 Ma Interplay between the Galápagos Hotspot and the Central American Subduction Zone: Kiel, Germany, Christian-Albrechts Universität, tesis doctoral, 102.

Liu, L., Spasojevic, S., Gurnis, M., 2008, Reconstructing Frallon Plate Subduction Beneath North America Back to the Late Cretaceous: Science, 322, 934-938. 
López, A., Sierra, G., Ramírez, D., 2006, Vulcanismo Neógeno en el suroccidente Antioqueño y sus implicaciones tectónicas: Boletín de Ciencias de la Tierra, 16, 1-42. ISSN 0120 3630, <http://www.scielo.org.co/pdf/bcdt/ $\mathrm{n} 19 / \mathrm{n} 19 \mathrm{a} 02 . \mathrm{pdf}>$.

López, E., 2009, Evolution tectono-stratigraphique du double bassin avant - arc de la marge convergente Sud Colombienne - Nord Equatorienne pendant le Cénozoïque: Niza, Francia, Université de Nice Sophia Antipolis, 371 pp.

Maloney, K.T., Clarke, G. L., Klepeis, K.A., Quevedo, L., 2013, The Late Jurassic to present evolution of the Andean margin: Drivers and the geological record: Tectonics, 32, 1-7.

Martinod, J., Husson, L., Roperch, P., Guillaume, B., Espurt, N., 2010, Horizontal subduction zones, convergence velocity and the bulding of the Andes: Earth y Planetary Science Letters , 299, 299-309.

McCourt, W., Aspend J.A., Brook, M., 1984, New geological and geochronological data from the Colombian Andes: continental growth by multiple accretion: Geological Society of London, 141, 831-845.

Montes, C., Bayona, G., Cardona, A., Buchs, D., Silva, C., Morón, S.E., Hoyos, N., Ramírez, D.A., Jaramillo, C., Valencia, V., 2012, Arc-Continent Collision and Orocline Formation: closing of the central American Seaway: Journal of Geophysical Research, 117, B04105.

Montes, C., Rodríguez-Corcho, A.F., Bayona, G., Hoyos, N., Zapata, S., Cardona A., 2019, Continental margin response to multiple arc-continent collisions: The northern Andes-Caribbean margin: Earth-Science Reviews, 198, 102903, doi.org/10.1016/j.earscirev.2019.102903.

Noriega-Londoño, S., Restrepo-Moreno, S.A, Vinasco, C., Bermúdez, M.A., Min, K., 2019. Thermochronologic and geomorphometric constraints on the Cenozoic landscape evolution of the Northern Andes: Northwestern Central Cordillera, Colombia: Geomorphology, doi: 10.1016/j. geomorph.2019.106890.

Núñez, A., 1981, Plutonismo terciario afectando el Batolito de Ibagué, en III Congreso Colombiano de Geología: Medellín, Colombia, Instituto Colombiano de Geología y Minería (INGENOMINAS), Libreto de resúmenes, $40 \mathrm{pp}$.

Osmundsen, P.T., Ebbing, J., 2008, Styles of extension offshore mid-Norway and implications for mechanisms of crustal thinning at passive margins: Tectonics, 27, 1-25, doi:10.1029/2007TC002242.

Ordóñez-Carmona, O., Restrepo, J.J., de Brito, R.S.C., Martens, U., Rodrigues, J.B., 2011. The late Paleocene age of the Santa Bárbara Batholith (Valle Colombia) and its implications on the evolution of the Arquía Complex, en XIV Congreso Latinoamericano de Geología - XIII Congreso Colombiano de Geología: Medellín, Colombia, Geología Colombiana, Memorias, 109.

Pardo-Casas, F., Molnar, P., 1987, Relative motion of the Nazca (Farallon) and south American plates since Late Cretaceous time: Tectonics, 6(3), 233-248.

Peccerillo, A., Taylor, S.R., 1976, Geochemistry of eocene calc-alkaline volcanic rocks from the Kastamonu area, Northern Turkey, Contributions to Mineralogy and Petrology, 58, 63-81. doi: 10.1007/BF00384745.

Pindell, J.L., Kennan, L., 2009, Tectonic evolution of the Gulf of Mexico, Caribbean and northern South America in the mantle reference frame: an update: Geological Society, London, Special Publications, 328, 1-55.

Pindell, J.L., Kennan, L., Maresch, W.V., Stanek, K.-P., Draper, G., Higgs, R., 2005, Plate kinematics and crustal dynamics of circum-Caribbean arc-continent interactions: tectonic controls on basin development in proto-Caribbean margins, en Ave Lallemant, H.G., Sisson, V.B. (eds.), Caribbean-South American Plate Interactions, Venezuela: Geological Society of America Special Paper, 394, 7-52.

Profeta, L., Ducea, M.N., Chapman, J.B., Paterson, S.R., Henriquez, S.M., Kirsch, M., Petrescu, L., DeCelles, P., 2015, Quantifying crystal thickness over time in magmatic arcs: Scientific Reports, 5:17786.

Ramos, V., 2009, Anatomy and global context of the Andes: Main geologic features and the Andean orogenic cycle: The geological Society of America memoir, 204, 31-65, DOI: https://doi.org/10.1130/2009.1204(02).

Restrepo, J.J., Toussaint, J.F., 2020. Tectonostratigraphic terranes in Colombia: An update. First part: Continental terranes, en Gómez, J., Mateus-Zabala, D. (eds.), The Geology of Colombia, Volume 1 Proterozoic - Paleozoic: Bogotá, Colombia, Servicio Geológico Colombiano, Publicaciones Geológicas Especiales 35, 27. https://doi.org/10.32685/pub.esp.35.2019.03.

Restrepo, J.J., Toussaint, J.F; González, H; Cordani, U.; Kawashita, K; Linares, E; Paria, C., 1991, Precisiones geocronológicas sobre el occidente colombiano, en Simposio Geológico Magmatismo andino y su marco tectónico:
Manizalez, Colombia, Universidad de Caldas, Memorias, Tomo 1, 1-21.

Restrepo, S., Foster, D., Stockli, D., Parra-Sánchez, L., 2009, Long-term erosion and exhumation of the "Altiplano Antioqueño", Northern Andes (Colombia) from apatite (U-Th)/He thermochronology: Earth and Planetary Science Letters, 278, 1-12.

Ribes, C., Manatschal, G., Ghienne, J.F., Karner, G., Johnson, C., Higueredo, P., Incerpi, N., Epin, M.E. 2019, The syn-rift stratigraphic record across a fossil hyper-extended rifted margin: the example of the northwestern Adriatic margin exposed in the Central Alps: International Journal of Earth Sciences, 108, 2071-2095, doi: 10.1007/s00531-019-01750-6.

Saenz, E., Paucar, C., Restrepo, J., 1996, Estudio de la evolución térmica del Batolito Antioqueño por huellas de fisión, en VII Congreso Colombiano de Geología, Bogotá, Colombia, Instituto Colombiano de Geología y Minería (INGENOMINAS), Memorias 2, 240-251.

Schellart, W.P., 2008a, Overriding plate shortening and extension above subduction zones: A parametric study to explain formation of the Andes Mountains: GSA Bulletin, 120, 11/12, 1441-1454. doi: 10.1130/B26360.1.

Schellart, W.P., 2008b, Subduction zone trench migration: Slab driven or overriding-plate-driven?: Physics of the Earth and Planetary Interiors, 170, 73-88.

Sdrolias, M., Müller, D., 2006, Controls on back-arc basin formation: Geochemistry Geophysics Geosystems, 7(4), 1-40.

Sillitoe, R., Jarmillo, L., y Muhammed, D., 1982, Setting, characteristics, and age of the andean porphyry copper belt in Colombia: Economic Geology, 77, $1837-1850$.

Somoza, R., Chidella, M., 2005, Convergencia en el margen occidental de América del sur durante el Cenozoico: Subducción de las placas de Nazca, Farallón y Aluk: Revista de la Asociacion Geológica Argentina, 60(4), 797-809.

Spera, F.J., 2000, Physical properties of magmas, en Sigurdsonn, H., (ed.), Encyclopedia of Volcanoes: New York, EE.UU., Academic Press, 171-190.

Spikings, R., Cochrane Ryan, Villagomez, D., Van Der, R., Vallejo, C., Winkler, W., Beate, B., 2014, The geological history of northwestern South America: from Pangaea to the early collision of the Caribbean Large Igneous Province (290-75 Ma): Gondwana Research, 27, 95-139.

Stolper, E., Walker, D., 1980, Melt density and the average composition of basalt: Contributions to Mineralogy and Petrology, 74, 7-12.

Vallejo, F., Salazar, A., Toro, L., 2011, Petrografía y geoquímica de las rocas intrusivas aflorantes entre los municipios de Mistrató y Belén de Umbría (Departamento de Risaralda, Cordillera Occidental Colombiana): Boletín de Geología, 33(2), 47-57.

Villagómez, D., Spikings, R., 2013, Thermochronology and tectonics of the Central and Western cordilleras of Colombia: Early Cretaceous- Tertiary evolution of the Northern Andes: Lithos, (160-161), 228-249.

Villagómez, D., Spikings, R., Magna, T., Kemmer, A., Winkler, W., Beltrán, A., 2011, Geochronology, geochemistry and tectonic evolution of the Western and Central cordilleras of Colombia: Lithos, 125, 875-896.

Whattam, S.A., Stern, R., 2014, Late Cretaceous plume-induced subduction initiation along the southern margin of the Caribbean and NW South America: The first documented example with implications for the onset of plate tectonics: Gondwana Research, 27, 38-63.

Whitmarsh, R., White, S., Horsefield, S.J., Sibuet, J.C., Recq, M., Louvel, V., 1996, The ocean-continental boundary off the western continental margin of Iberia: Crustal structure west of Galicia Bank: Journal of Geophysical Research, 101(B12), 28,291-28,314.

Zapata-García, G., Rodríguez-García, G., 2020, New contributions to the knowledge about the Chocó-Panamá Arc in Colombia, including a new segment south of Colombia, en Gómez, J., Mateus-Zabala, D. (eds.), The Geology of Colombia, Volume 3: Paleogene-Neogene. Servicio Geológico Colombiano, Publicaciones Geológicas Especiales 37, 32 pp. Bogotá, Colombia, doi:10.32685/pub.esp.37.2019.14.

Zhang, C., Su., M., Pang, X., Zheng, J., Liu, Z., Sun, G., Manatschal, G., 2019, Tectono-sedimentary analysis of the hyper-extended Liwan sag basin (mid-northern margin of the South China Sea): Tectonics, 38, 22, doi: $10.1029 / 2018$ TC005063.

Manuscrito recibido: marzo 4, 2020

Manuscrito corregido recibido: septiembre 21, 2020

Manuscrito aceptado: octubre 8, 2020 